\title{
2. Die arbeitsrechtlichen Veränderungen
}

\section{Gesetz der Arbeit}

Mit der Gründung der DDR im Oktober 1949 sowie dem langsam einsetzenden Wechsel der Instrumentarien bei der Arbeitskräftelenkung, der zum Teil schon ein Jahr zuvor begonnen hatte, stellte sich die Frage, inwieweit die arbeitsrechtlichen Bestimmungen, die zu einem erheblichen Teil in den ersten beiden Nachkriegsjahren festgelegt worden waren, dieser Entwicklung angepaßt werden mußten. Hinzu kam die Tatsache, daß die SKK ${ }^{1}$ als Nachfolgeorganisation der SMAD einige Befehle aus der Zeit zwischen 1945 und 1949 selber aufhob². Außerdem ersetzten der Ministerrat bzw. die einzelnen Ministerien in enger Absprache mit der Besatzungsmacht sowjetische Befehle durch deutsche Verordnungen ${ }^{3}$. Walter Ulbricht kündigte in seiner Funktion als Stellvertreter des Ministerpräsidenten gegenüber dem Vorsitzenden der SKK, Armeegeneral Wassili I. Tschujkow, die Ausarbeitung einiger sozialpolitischer Gesetze an: So bereitete die deutsche Seite die Veröffentlichung eines Arbeitsgesetzes vor, „in welche[m] die Bestimmungen des Befehls 234 und anderer Befehle, die die Arbeitsfragen betreffen, aufgenommen werden" 4 . Darüber hinaus befanden sich Gesetze zum Gesundheitswesen, zur „Hilfe für die Umsiedler“, über „Kulturfragen“ sowie zur „Durchführung der verfassungsmäßigen Bestimmungen über die Rechte der Frauen" in Planung.

Das sogenannte Arbeitsgesetz war von Anfang an als Rahmengesetz konzipiert, das grundlegende Bestimmungen zu zahlreichen arbeitsrechtlichen Fragen enthalten sollte: Diese reichten vom Mitbestimmungsrecht bis hin zu Arbeitszeitregelungen sowie zur Gesundheitsfürsorge ${ }^{5}$. Die SED-Führung und das Arbeitsministerium waren sich offensichtlich darüber einig, daß das geplante Gesetz im weiteren Verlauf durch Verordnungen und Durchführungsbestimmungen „in die praktische Wirklichkeit" umzusetzen war'. Die Fertigstellung des Gesetzestextes stand unter einem großen Zeitdruck: Aus Anlaß des 60. Jahrestages der Feierlichkeiten zum 1. Mai sollte das Arbeitsgesetz in Kraft treten. Da jedoch die juristischen Verfeinerungen in Form von Verordnungen zu den einzelnen Bereichen des Arbeitsrechts bereits vorgesehen waren, wog dieses Manko aus Sicht der beteiligten Stellen nicht sonderlich schwer. Inwieweit die sowjetische Besatzungsmacht ihrerseits eine rasche Veröffentlichung verlangte, läßt sich nur erahnen.

Ein erster Entwurf, der sehr skizzenhaft gehalten und vermutlich vom Ministerium für Arbeit und Gesundheitswesen erstellt worden war, schrieb eingangs das Recht auf Arbeit fest und verknüpfte dies allerdings mit der „Verpflichtung, seine

1 Vgl. Das SKK-Statut.

2 Eine von der SKK angefertigte Liste enthielt insgesamt 13 Befehle, unter anderem den SMAD-Befehl Nr. 65 vom 15. 9. 1945. SAPMO, NY 4182/1194, Bl. 61 f.

3 Auch dazu legte die SKK eine Liste vor, die 54 Befehle enthielt, die bis zur Veröffentlichung von entsprechenden Bestimmungen und Verordnungen der DDR-Regierung in Kraft blieben, darunter auch der SMAD-Befehl Nr. 153 vom 29. 11. 1945. SAPMO, NY 4182/1194, Bl. 63-66.

+ Ebenda, Bl. 72, Ulbricht am 12. 2. 1950 an Tschujkow.

5 SAPMO, NY 4090/564, Bl. 1, Staatssekretär Peschke am 23. 3. 1950 an das Büro des Ministerpräsidenten (Tzschorn), S. 1.

6 Ebenda, S. 2. 
Arbeitskraft für das gemeinsame Wohl einzusetzen"7. Ein eigenständiges Kapitel über die "planmäßige Verwendung der Arbeitskräfte“ brachte keinerlei Veränderungen: Das Ministerium für Arbeit und Gesundheitswesen und die Fachministerien hatten nach wie vor die Aufgabe, der Grundstoff- und Schwerindustrie Arbeitskräfte zur Verfügung zu stellen. Besonders hervorgehoben wurde dabei der Bergbau. Die Frauen, die noch nicht erwerbstätig waren, sollten verstärkt für die Teilarbeitsmärkte mobilisiert werden. Um dieses Ziel zu erreichen, war die "Schaffung von Kindergärten und andere[n] soziale[n] Einrichtungen“ geplant ${ }^{8}$. Diese Maßnahmen zur Gewinnung neuer Arbeitskraftreserven wurden ergänzt durch die Vorgabe der Steigerung der Arbeitsproduktivität in den Betrieben. Durch eine verstärkte Mechanisierung sollte die betriebliche Arbeitsleistung verbessert werden, was letztlich die Entlassung von Arbeitern zur Folge hatte. Soziale Maßnahmen zur Abfederung dieses Prozesses waren zu diesem Zeitpunkt noch nicht vorgesehen; das Arbeitsministerium hatte offensichtlich noch nicht einmal ein entsprechendes Problembewußtsein entwickelt. Die Erfahrungen, die im Zusammenhang mit den beiden Sofortprogrammen im Laufe des Jahres 1949 gesammelt werden konnten, hätten freilich ausreichend Anlaß zur Skepsis geboten.

Wenig später lag ein ausführlich gehaltener Gesetzentwurf vor, der bereits die endgültige Überschrift trug: Gesetz „zur Förderung und Pflege der Arbeitskräfte, zur Steigerung der Arbeitsproduktivität und zur Verbesserung der materiellen und kulturellen Lage der Arbeiter und Angestellten "9. Die Präambel reflektierte den sich stark verschärfenden Ost-West-Gegensatz deutlich: So betonten die Verfasser, daß in der DDR „durch die Entfaltung der Industrie, der Landwirtschaft und des Verkehrs neue Arbeitskräfte geworben“ werden müßten. Dagegen steige die Arbeitslosigkeit ,in dem von den westlichen Monopolisten besetzten Deutschland unaufhörlich" 10 . Die propagandistische Instrumentalisierung wurde auch beim ersten Kapitel unverkennbar, in dem das Recht auf Arbeit proklamiert wurde. Die darin ebenfalls enthaltene Arbeitspflicht ging fast unter. Die anschließenden Kapitel beschäftigten sich mit dem Mitbestimmungsrecht, der Steigerung der Arbeitsproduktivität, der Förderung der Aktivisten- und Wettbewerbsbewegung, dem Kündigungsrecht und dem Arbeitsschutz sowie der sogenannten Verbesserung der materiellen und kulturellen Lage der Arbeiter und Angestellten. Von zentraler Bedeutung für unseren Untersuchungsgegenstand waren vor allem die Kapitel V („Planmäßige Verwendung der Arbeitskräfte“) und VI („Heranbildung von fachlichem Nachwuchs und Qualifizierung von Frauen“) ${ }^{11}$. Inhaltlich orientierte sich der Gesetzentwurf weitgehend an dem bereits vorgestellten Konzeptpapier: Er machte vor allem die gestiegene Bedeutung der beruflichen Ausund Weiterbildung für die Planungsbehörden deutlich, nachdem sich die Mobilisierung zusätzlicher Arbeitskräfte als nicht sehr erfolgversprechend herausgestellt

\footnotetext{
SAPMO, NY 4090/564, Bl. 9-11, hier Bl. 9, Grundzüge des Gesetzentwurfes für das Arbeitsgesetz (o.Verf., o.D.)

$\&$ Ebenda, Bl. 10.

9 SAPMO, NY 4090/564, Bl. 22-39. Der Entwurf stammte allem Anschein nach vom Ministerium für Arbeit und Gesundheitswesen.

10 Ebenda, Bl. $22 \mathrm{f}$.

11 Ebenda, Bl. 31-33.
} 
hatte. Diese Verlagerung der arbeitsmarktpolitischen Instrumente hatte sich aber schon vor der DDR-Gründung angedeutet und trat nunmehr immer stärker in den Vordergrund.

Justizminister Max Fechner berichtete am 31. März, daß der Gesetzentwurf noch vor dem 1. Mai durch das Ministerium für Arbeit und Gesundheitswesen fertiggestellt werden müsse, wobei sein Ministerium hinzugezogen werden solle ${ }^{12}$. Der Zeitdruck war somit in Absprache mit dem Ministerrat und der SKK erhöht worden. Die Feinabstimmung mußte demnach - wie vorgesehen - weiteren Verordnungen überlassen bleiben. Mit der Ausarbeitung des endgültigen Entwurfes beauftragte das Politbüro eine Kommission mit Vertretern des ZK-Apparates der SED, des FDGB-Bundesvorstandes sowie des Arbeitsministeriums. Zu diesem Redaktionsgremium gehörten Willi Stoph, Max Herm, Fritz Selbmann, Alexander Starck, Paul Peschke und Margarete Wittkowski ${ }^{13}$. Gleichzeitig legte die SEDFührung die inhaltliche Ausrichtung fest: „Das Gesetz muß auf der Linie unserer Wirtschaftspolitik aufgebaut sein. "14 Damit wurden die einzelnen sozialpolitischen Bestandteile der allgemeinen Wirtschaftsplanung und -lenkung klar untergeordnet. An der Gliederung des Entwurfes änderte sich kaum etwas. Es wurde nur ein weiteres Kapitel neu aufgenommen, das sich explizit der Neuregelung der Sozialversicherung widmen sollte. Dieser Themenkomplex fand allerdings im veröffentlichten Gesetzestext keine Berücksichtigung mehr. Nach den Vorstellungen der Politbüromitglieder hatte das Arbeitsgesetz "genaue Termine für den Erlaß der Durchführungsbestimmungen und die entsprechenden Strafbestimmungen [zu] enthalten " ${ }^{15}$. Auf diese Weise sollte offensichtlich eine erhebliche zeitliche Verzögerung bei der Ausarbeitung der weiteren arbeitsrechtlichen Bestimmungen vermieden werden. Abschließend erteilte das Politbüro dem FDGBBundesvorstand und der ZK-Abteilung Massenagitation den Auftrag, "sofort einen Plan für die Entfaltung der gewerkschaftlichen und allgemeinen Agitation auszuarbeiten".

Während die Ausarbeitung des Gesetzes der Arbeit eine Angelegenheit der SED-Führung, des DDR-Ministerrates sowie der beteiligten Ministerien (vor allem: Arbeitsministerium, Ministerium für Planung) war, wurden zur „Popularisierung" des Gesetzes Vertreter der Gewerkschaften sowie der Betriebe mit eingebunden. Ministerpräsident Otto Grotewohl lud aus diesem Anlaß zu einer "gemeinsamen Aussprache" in das Gästehaus der Regierung am 11. April 1950 ein ${ }^{16}$. $\mathrm{Zu}$ diesem Zeitpunkt waren die Arbeiten am Gesetzentwurf schon längst abgeschlossen, und der Ablauf der Volkskammersitzung, auf der das Gesetz offiziell verabschiedet werden sollte, war bereits festgelegt worden. Das Politbüro hatte auf seiner Sitzung am 11. April dem überarbeiteten Entwurf „grundsätzlich“ zugestimmt und nur noch kleinere Veränderungen vorgenommen, die jedoch die

12 SAPMO, NY 4090/563, Bl. 64, Notiz des Sekretariats des Ministerpräsidenten (Reckow) vom 4. 4. 1950.

13 SAPMO, DY 30/IV 2/2/81, Bl. 23f., hier Bl. 23, Protokoll der Sitzung des Politbüros am 4. 4. 1950.

14 Ebenda.

15 Ebenda, Bl. 24.

is SAPMO, NY 4090/564, Bl. 64, Persönlicher Referent des Ministerpräsidenten Tzschorn am 5. 4. 1950 an den Minister für Planung Rau. 
Abschnitte zur Arbeitskräfteplanung und Nachwuchsförderung nicht betrafen ${ }^{17}$. Zwei Tage später beschloß die DDR-Regierung einstimmig, den Entwurf in der Volkskammer einzubringen. Ministerpräsident Grotewohl erhielt die Aufgabe, dort eine ausführliche Begründung abzugeben ${ }^{18}$. Die Beratungen in der Volkskammer konnten rasch beendet werden. Über die Notwendigkeit, das Rahmengesetz in Kraft treten zu lassen, herrschte Einmütigkeit. Staatsminister Peschke wies bei einer gemeinsamen Sitzung des Ausschusses für Arbeit und Gesundheitswesen sowie des Wirtschaftsausschusses auf den akut bestehenden Arbeitskräftemangel erneut hin: „Die Arbeiter kommen nicht mehr von selbst auf das Arbeitsamt oder in die Betriebe gelaufen und bieten ihre Arbeitskraft an." ${ }^{19} \mathrm{Um}$ die aufgestellten Wirtschaftspläne aber erfüllen zu können, müsse man sich - so Peschke weiter - „sehr ernste Sorgen“ über die Rekrutierung von Arbeitern machen. Der Staatssekretär im Arbeitsministerium sprach dem Gesetzesvorhaben Signalwirkung für die junge Bundesrepublik zu und ging davon aus, daß es längere Zeit Bestand haben werde: „Es handelt sich also nicht um ein Gesetz, das nur heute und für einige Zeit Gültigkeit hat, sondern um einen Gesetzentwurf, der in seinen Grundzügen die Möglichkeit schafft, die Entwicklung einer ganzen Periode vor uns zu gestalten. " 20 Peschke bezeichnete das vorliegende Gesetz sogar als "Grundgesetz der Arbeit" ${ }^{21}$. Noch deutlicher war Grotewohl am 19. April vor der Volkskammer, als er betonte, es käme nicht darauf an, „eine Anhäufung formaler Paragraphen und Bestimmungen zu verankern, sondern als ein Rahmengesetz alle einschlägigen Bestimmungen zu umfassen und sie mit einer solchen Elastizität auszustatten, daß es sowohl dem Parlament wie auch der dem Parlament verantwortlichen Regierung jederzeit möglich ist, das Arbeitsrecht den sich stets wandelnden Formen [des] Wirtschaftslebens anzupassen" 22 . Bei den Ausschußberatungen am 14. und 19. April wurden keine grundsätzlichen Einwände bzw. Verbesserungsvorschläge vorgebracht ${ }^{23}$, so daß die Volkskammer den Entwurf am 19. April beraten und beschließen konnte. Damit wurde allerdings der Mitarbeit dieses Gremiums beim Gesetzgebungsverfahren nur formal Rechnung getragen. Entscheidende Veränderungen konnten nicht mehr vorgebracht werden, wobei auch darauf hingewiesen werden muß, daß die Volkskammermitglieder ihrerseits auf eigene Initiativen verzichteten. Das Gesetz der Arbeit wurde am 28. April im Gesetzblatt veröffentlicht und konnte, wie anfangs vorgesehen, am 1. Mai 1950 in Kraft treten ${ }^{24}$. Die Schlußbestimmung des Arbeitsgesetzes legte wiederum fest, daß bisher bestehende arbeitsrechtliche Bestimmungen, die dem neuen Gesetz widersprachen, automatisch außer Kraft traten ${ }^{25}$. Ausgenommen

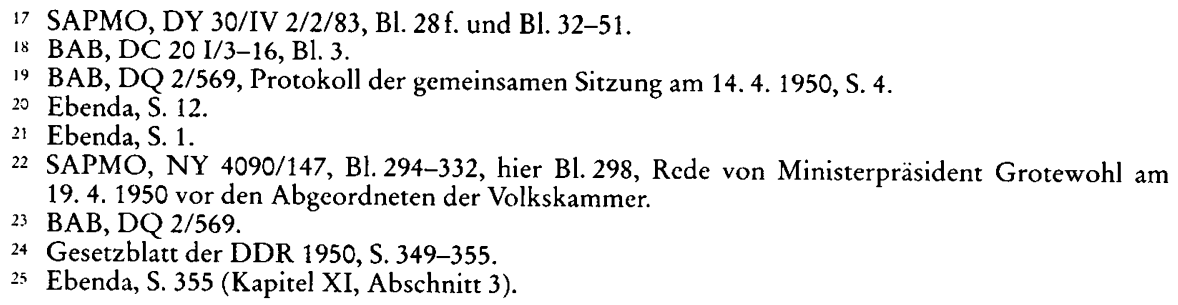


blieben jedoch sowjetische Befehle, die nur von der SKK aufgehoben werden konnten ${ }^{26}$.

Das Gesetz der Arbeit hatte das bereits zuvor geltende Prinzip der Bereitstellung von Arbeitskräften für die „Schwerpunkte der Wirtschaft“ bestätigt. Dieses Ziel sollte nach wie vor auf dem Wege der Anwerbung realisiert werden; von Zwangsmaßnahmen war keine Rede. Auffallend ist die Tatsache, daß bereits wenige Wochen nach Inkrafttreten des Gesetzes von einzelnen Arbeitsämtern die Forderung erhoben wurde, Strafbestimmungen zur Durchsetzung der Lenkungsmaßnahmen einzuführen. So schlug beispielsweise das Amt für Arbeit und Sozialfürsorge Bitterfeld vor, Geldstrafen zwischen 50 und $10000 \mathrm{DM}$ zu verhängen, falls Arbeitgeber bzw. Betriebsleiter Einstellungen oder Entlassungen vornehmen, ohne vorab das zuständige Arbeitsamt einzuschalten ${ }^{27}$. Damit deutete sich ein alter Konflikt zwischen der Arbeitsverwaltung auf der einen und den Betrieben auf der anderen Seite wieder an. Selbst der Einsatz von Zwangsmaßnahmen hatte vor 1949 nicht zu der erhofften Reduzierung der zwischenbetrieblichen Fluktuation geführt. Die einzelnen Betriebe ließen es sich nicht nehmen, für die Abdeckung des Arbeitskräftebedarfs selber zu sorgen und dies nicht nur dem zuständigen Arbeitsamt zu überlassen. Die im Sommer 1951 in Kraft tretende Verordnung, die im Anschluß an diesen Abschnitt ausführlicher vorgestellt wird, bestätigte letztlich diese Praxis und verabschiedete sich endgültig von arbeitsmarktpolitischen Instrumenten, die primär auf $Z$ wangsmaßnahmen begründet waren. Darüber hinaus sah die am 7. Juli 1951 in Kraft getretene Verordnung ebenfalls keine Beteiligung der Arbeitsämter bei Kündigungen mehr vor ${ }^{28}$. Betriebliche Entlassungen wurden zukünftig von der Zustimmung der Betriebsgewerkschaftsleitungen abhängig gemacht. Für den betroffenen Arbeiter bzw. Angestellten bestand auch nach wie vor die Möglichkeit, das zuständige Arbeitsgericht einzuschalten.

\section{Verordnung über die Aufgaben der Arbeitsverwaltungen und über die Lenkung der Arbeitskräfte}

Der Aufbau der Arbeitsverwaltung hatte sich, wie gezeigt werden konnte, unter dem Eindruck der prinzipiellen Auseinandersetzung zwischen den Vorstellungen einer Kommunalisierung oder einer Zentralisierung verzögert und nicht unerhebliche Reibungsverluste hervorgerufen. Mit dem Aufbau einer zentralen Wirtschaftsverwaltung in Form der DWK und des Übergangs zur Planwirtschaft konnte diese Frage letztlich entschieden werden. Obwohl die hierachische Struktur mit der HVAS an der Spitze durchgesetzt wurde, tauchten im Laufe des Sommers 1949 erste Pläne zur Eingliederung der Ämter für Arbeit und Sozialfürsorge

26 Darauf wicsen SKK-Vertreter in einer Stellungnahme zum Arbeitsgesetz ausdrücklich hin. Vgl. SAPMO, NY 4182/1090, Bl. 133-138, hier Bl. 138. Aus der beiliegenden SED-Hausmitteilung Stophs vom 24. 4. 1950 geht hervor, daß es sich um eine sowjetische Stellungnahme handeln muß. Vgl. ebenda, Bl. 132.

27 LA Magdeburg LHA, Rep. K MW, Nr. 10185, Bl. 334, Amt für Arbeit und Sozialfürsorge Bitterfeld am 8.6. 1950 an das Ministerium für Arbeit und Gesundheitswesen des Landes SachsenAnhalt.

28 Verordnung über Kündigungsrecht, in: Gesetzblatt der DDR 1951, S. 550 f. 
in die Kommunalverwaltung auf, die von der HVAS vorgelegt wurden ${ }^{29}$. Angesichts der vorherigen Auseinandersetzung überraschte dieser Schritt. Bei näherer Betrachtung der ersten Entwürfe wird jedoch die eigentliche Stoßrichtung deutlich: Diese zielte nämlich auf eine Verbesserung der Zusammenarbeit der unterschiedlichen Verwaltungsebenen bei der Arbeitskräftelenkung. Nach den Vorstellungen der HVAS sollten die neu strukturierten Arbeitsämter nach wie vor der zentralen Anleitung und Kontrolle unterworfen bleiben. Auffallend ist die Umgehung der zuständigen Landesverwaltungen, deren Zuständigkeiten im Anordnungsentwurf überhaupt nicht thematisiert wurden. Den Landesregierungen blieb nur das Recht vorbehalten, einzelne Kreise vorzuschlagen, bei denen die Verwaltungsreform als erstes durchgeführt werden sollte. Die Pläne zu einer Verwaltungsstrukturreform verband die HVAS mit Überlegungen, ihre arbeitsmarktpolitischen Maßnahmen an die Erfordernisse der allgemeinen Wirtschaftsplanung anzupassen.

Die Abteilung I a des Ministeriums für Arbeit und Gesundheitswesen legte Ende 1949 einen Verordnungsentwurf vor, der Bezug nahm auf Artikel 139 der DDR-Verfassung und die Landesministerien in die neue Verwaltungsstruktur stärker einzubinden versuchte ${ }^{30}$. Die herausgehobene Position des Berliner Ministeriums blieb jedoch unangetastet: Diese erstreckte sich sowohl auf Personal- wie Sachfragen. Die Landesregierungen zeigten sich aber mit den Bestimmungen der geplanten Verordnung nicht völlig einverstanden und verlangten ein größeres Mitspracherecht bei der Stellenbesetzung, die sich nicht nur auf die Leitung der neu zu bildenden Abteilung Arbeit bei den Stadt- und Landkreisen, sondern auf sämtliche leitenden Funktionen beziehen müßte ${ }^{31}$. Ein Alternativentwurf der sächsischen Landesregierung klammerte diese personalpolitische Kontroverse zunächst einmal aus ${ }^{32}$ : Demzufolge sollte die Mitwirkung des zuständigen Landesamtes in dieser Frage durch weitere Anordnungen geregelt werden. Bei der Auswahl der Kreise, in denen die Verwaltung neu strukturiert werden sollte, sah der sächsische Entwurf vor, den Einfluß des Ministeriums für Arbeit und Gesundheitswesen zurückzudrängen: Dies hatten demnach die Landesministerien im Einvernehmen mit dem Ministerium des Innern zu entscheiden.

Bereits Ende Januar 1950 fand eine erste Sitzung statt, auf der Vertreter des Ministeriums für Arbeit und Gesundheitswesen mit den zuständigen Abteilungsleitern der Länder über den Funktionswandel der Arbeitskräftelenkung berieten. Staatssekretär Paul Peschke wies bei der Gelegenheit zum wiederholten Male darauf hin, daß die Erfüllung der aufgestellten Produktionspläne nur möglich sei, „wenn die entsprechenden Arbeitskräfte rechtzeitig zur Verfügung gestellt werden" ${ }^{33}$. Die Methoden der Arbeitsvermittlung seien daher nicht mehr ausreichend. Mit dem Rückgang der Arbeitslosenzahlen hätten sich - so Peschke weiter - die Aufgaben grundsätzlich verändert. Die von den Statistiken erfaßten Er-

$29 \mathrm{BAB}, \mathrm{DQ} 2 / 1754$, Anordnungsentwurf der HVAS-Abt. I a (o.D.).

$30 \mathrm{BAB}, \mathrm{DQ}$ 2/1734, Verordnungsentwurf des Ministeriums für Arbeit und Gesundheitswesen (Abt. I a) vom 26. 12. 1949.

31 BAB, DQ 2/1754, Ministerium für Arbeit und Gesundheitswesen der Landesregierung Mecklenburg am 29. 12. 1949 an das Ministerium für Arbeit und Gesundheitswesen.

$32 \mathrm{BAB}, \mathrm{DQ} 2 / 1734$, Entwurf der sächsischen Landesregierung (o.Verf., o.D.).

33 BAB, DQ 2/1152, Protokoll über die Sitzung am 24. 1. 1950, S. 1. 
werbslosen müßten in die Arbeitswelt zurückgeführt werden: „Was sich heute als arbeitslos [...] ausweist, sind ,Zugvögel', $d$.h. Leute, die weglaufen, um einen besseren Platz zu suchen. Das darf es in unserer Wirtschaft nicht mehr geben. Dagegen muß seitens der Betriebe und seitens der Ämter für Arbeit gekämpft werden. “34 Es war vermutlich das Ziel dieser Tagung, einen Alleingang der Länder zu verhindern und die Tätigkeit der Arbeitsverwaltung auf zentraler Ebene mit der Länderebene besser abzustimmen. So äußerten Vertreter des Ministeriums für Arbeit und Gesundheitswesen ihr Mißfallen über Absprachen zwischen den Landesverwaltungen, die offensichtlich mit dem Berliner Ministerium nicht abgestimmt worden waren, und erhoben sogar den schwerwiegenden Vorwurf der Fraktionsbildung. Nach einer kurzen, kontrovers geführten Debatte wurde im Protokoll festgehalten, daß zukünftig "Ländersitzungen ohne Anwesenheit des Berliner Ministeriums nicht angängig sind" 35 .

Die Arbeitsverwaltung arbeitete parallel an zwei getrennten Entwürfen: jeweils ein Gesetz bzw. eine Verordnung über die inhaltliche Ausrichtung der Arbeitskräftelenkung sowie zur Verwaltungsreform. Erst zu einem späteren Zeitpunkt wurden beide Vorhaben zu einem Verordnungsentwurf zusammengefaßt. Anfang 1950 arbeitete die Abteilung I a des Ministeriums für Arbeit und Gesundheitswesen einen Gesetzentwurf „über die Erfassung und Lenkung der Arbeitskräfte“ aus, der jedoch nur die bereits geltenden arbeitsrechtlichen Bestimmungen für die Vermittlung von Arbeitskräften zusammenfaßte ${ }^{36}$. So wurden wesentliche Bestandteile der Verordnung vom 2. Juni 1948 übernommen, unter anderem die Abschnitte zur Arbeitseinweisung. Dies überraschte umso mehr, da dieses Zwangsmittel in der Praxis kaum noch Anwendung gefunden hatte. Offensichtlich wollte die Arbeitsverwaltung diese Möglichkeit im Hinblick auf die ungewissen Anforderungen der sowjetischen Besatzungsmacht nicht ohne weiteres völlig ausschließen. Die Phase, in denen die Arbeitsämter mit Befehlen der SMAD und der SMA in den Ländern überhäuft worden waren, blieb somit in den Köpfen präsent und wirkte sehr wahrscheinlich immer noch prägend für die Tätigkeit der Wirtschaftsplaner.

Mit den Ländern konnte offensichtlich recht schnell eine Verständigung über die Neuordnung der Verwaltung erzielt werden: Auf einer Konferenz nahmen die Ländervertreter den vorgelegten Entwurf einstimmig an ${ }^{37}$. Nach Absprache mit der SKK galt zunächst der 30. Juni 1950 als Stichtag der Strukturreform, die auch zum Stellenabbau und somit zu Haushaltseinsparungen genutzt werden sollte. Aus der Tatsache, daß der Verordnungsentwurf vom 4. März ${ }^{38}$ keine wesentlichen inhaltlichen Veränderungen enthielt, kann gefolgert werden, daß die Landesämter auf eine weiterführende Beteiligung bei der Stellenbesetzung nicht bestanden hatten. Veränderungen ergaben sich nur bei der Festlegung der einzelnen Bereiche innerhalb der Abteilungen Arbeit bei den Stadt- und Landkreisen; deren Anzahl

34 Ebenda, S. 2.

35 Ebenda.

36 BAB, DQ 2/1766, B1. 506-510, Entwurf der Abt. I a (o.D.).

$37 \mathrm{BAB}, \mathrm{DQ} 2 / 2060$, Aktennotiz über eine Besprechung zwischen Mitarbeitern des Ministeriums des Innern und des Ministeriums für Arbeit und Gesundheitswesen am 28. 2. 1950, S. 2.

$38 \mathrm{BAB}, \mathrm{DQ} 2 / 1416$. 
schwankte nämlich zwischen vier und sechs. Obwohl bei der Ausarbeitung des Verordnungsentwurfes keinerlei Schwierigkeiten aufgetreten waren und der drohende Konflikt zwischen Ländern und DDR-Regierung vermieden werden konnte, verschob der Ministerrat die Beschlußfassung am 13. April ohne Angabe von Gründen ${ }^{39}$. Stimmungsberichte der Landesverwaltungen, die vermutlich vom Amt für Information kurz zuvor in Auftrag gegeben und anschließend gesammelt worden waren, zeichneten ein negatives Image der Arbeitsämter nicht nur bei den Betriebsleitern, sondern auch bei den Arbeitern. Während sich erstere dagegen aussprachen, den Arbeitsämtern größere Vollmachten zu gewähren, lehnten letztere die Arbeitsämter "heute noch wegen der Methoden in der Hitlerzeit ab“, so der Bericht, der sich vor allem auf Meldungen aus vier Großbetrieben stützte ${ }^{40}$. Gerade die geplanten Bestimmungen zur Arbeitseinweisung riefen allgemein Unmut hervor. Inwiefern diese Stimmungsberichte, die keineswegs repräsentativ waren, letztlich zu dieser zeitlichen Verzögerung beitrugen, kann nur vermutet werden.

Mit dem Beschluß des Ministerrates geriet die Fertigstellung der geplanten Verordnung zunächst ins Stocken. Das Ministerium für Arbeit und Gesundheitswesen ließ sich jedoch davon nicht beeindrucken und legte Anfang Juni 1950 einen weiteren Entwurf vor, der kaum Veränderungen enthielt und offensichtlich auch die Zustimmung des Ministeriums des Innern gefunden hatte ${ }^{41}$. Neu war dagegen nur der damit zusammenhängende Entwurf einer Ausführungsbestimmung, welche die Struktur der neuen Abteilungen festlegen sollte: Demzufolge waren vier Bereiche vorgesehen (Arbeitsproduktivität, Berufsausbildung und Umschulung, Arbeitskraftlenkung und Arbeitsschutz), deren Aufgabengebiete klar voneinander abgegrenzt wurden ${ }^{42}$. Auffallend ist, daß die Leitung des Ministeriums nahezu zeitgleich eine Überprüfung der Arbeitsämter in Auftrag gab43. Diesen Schritt hatte Staatssekretär Peschke am 9. Juni angeordnet. Dabei ergaben sich Differenzen mit Minister Steidle, der sich letztlich übergangen fühlte ${ }^{44}$. Bei einem Spitzengespräch am 14. Juni, das unter Vorsitz von Walter Ulbricht stattfand, und an dem unter anderem die Leitungen des Ministeriums für Arbeit und Gesundheitswesen und der Zentralen Kommission für Staatliche Kontrolle (ZKSK) teilnahmen, wurde die Reorganisation grundsätzlich bestätigt ${ }^{45}$. Staatssekretär Peschke, der bisher stets zu den Befürwortern einer Eingliederung der Arbeitsämter in die kommunale Selbstverwaltung gehört hatte, revidierte jedoch seine Position und zog den Verordnungsentwurf zurück. Er begründete diesen Meinungswechsel damit, daß sich die DDR-Regierung ansonsten „des zentralen Einflusses auf die sachlichen und materiellen Verhältnisse und der Arbeitsmethoden weitgehend

$39 \mathrm{BAB}, \mathrm{DC} 20 \mathrm{I} / 3-16, \mathrm{Bl} .3$.

$40 \mathrm{BAB}, \mathrm{DQ} 2 / 2095$, Bericht des Amtes für Information (HA Informationskontrolle) vom 17.4. 1950, S. 2. Bei den Großbetrieben handelte es sich um das Chemiewerk Leuna, die Maschinenfabrik Halle-Saale-Werke, die Elektromotorenfabrik Wernigerode sowie das Walzwerk Ilsenburg.

+1 BAB, DQ 2/906, Bl. 169 f., Verordnungsentwurf (handschriftlich: 9. 6. 50).

42 Ebenda, Bl. $171 \mathrm{f}$.

t3 BAB, DQ 2/906, Bl. 153, HA Arbeit am 13.6. 1950 an die Abteilungen des Hauses.

4 Ebenda, Bl. 151, Minister Steidle am 16.6. 1950 an Staatssekretär Peschke und Hauptabteilungsleiter Litke.

45 BAB, DC 1/1536, ZKSK-Vorsitzender Fritz Lange am 6. 7. 1950 an Staatssekretär Peschke. Dieser Beschluß ging offenbar auf einen entsprechenden Antrag der ZKSK zurück. 
[...] entäußer[t]" hätte ${ }^{46}$. Die Befriedigung des Arbeitskräftebedarfs mache - so Peschke weiter - „eine straffe Lenkung von zentraler Stelle aus notwendig“. Abschließend bat der Staatssekretär den ZKSK-Vorsitzenden, der sich bisher in arbeitsmarktpolitische Fragen kaum eingeschaltet hatte und auch nicht über entsprechende Erfahrungen verfügte, um Rat. Fritz Lange, der sich Ende der vierziger bzw. Anfang der fünfziger Jahre bei der rigorosen Zurückdrängung des privaten Wirtschaftssektors einen Namen gemacht hatte und dabei in enger Absprache mit Ulbricht sowie unter Ausschaltung des Justizwesens zahlreiche Wirtschaftsstrafprozesse durchführen ließ, die oftmals mit langjährigen Haftstrafen für die Angeklagten endeten ${ }^{47}$, ergriff diese Gelegenheit dankbar. Seine Reaktion war eindeutig: „Ich weiß wirklich nicht, was ich zu dieser Antwort sagen soll. Nach meiner Auffassung ist es eine glatte Bankrott-Erklärung." ${ }^{48}$

Das Ministerium für Arbeit und Gesundheitswesen beschränkte sich in der Folgezeit bei der Neuordnung der Arbeitsverwaltung auf die Neueinteilung bzw. Abgrenzung der geographischen Zuständigkeitsbereiche der einzelnen Arbeitsämter ${ }^{49}$. Dadurch sollte die Zusammenlegung von Stadt- und Landkreisen und damit eine Stelleneinsparung erreicht werden. Diesen Versuch hatte die DVAS/ HVAS bereits vor 1949 unternommen, allerdings mit begrenztem Erfolg. Der vom Hauptabteilungsleiter Litke ausgearbeitete Plan sah die Reduzierung der Anzahl der Arbeitsämter von 125 auf 55 vor. Von den Kürzungsvorschlägen waren vor allem die Länder Sachsen-Anhalt und Mecklenburg betroffen: Hier sollten nur noch ein Drittel bzw. ein Viertel der Ämter bestehen bleiben ${ }^{50}$. Die Untergliederung der Amtsbezirke in Neben- und Hilfsstellen sollte dagegen nach wie vor den Arbeitsämtern überlassen bleiben. Mit diesem Konzept war aber eine erhebliche Verringerung der Personal- und Sachkosten nicht zu erwarten, da die „einzuziehenden Hauptämter" automatisch als Nebenstellen weiterbestehen sollten.

Eine Reduzierung der Personalkosten konnte jedoch im Laufe des Jahres 1950 nicht mehr durch die angestrebte Verordnung erfolgen, da sich deren Veröffentlichung bis zum Sommer 1951 hinzog, sondern durch die Sparmaßnahmen, die 1949 für die Arbeitsämter angeordnet worden waren. Demzufolge waren 10 Prozent der anfallenden Kosten für Personal einzusparen. Diese Kürzungsanweisungen betrafen im übrigen auch andere Verwaltungszweige, so z.B. die Verwaltung der Sozialversicherung51, und wurden in der Frühphase der DDR häufig erteilt. Dadurch wurde die Verwaltungstätigkeit mitunter akut gefährdet bzw. nicht unerheblich eingeschränkt: So wies der brandenburgische Minister für Arbeit und Sozialwesen Karl Grobbel darauf hin, daß angesichts der ohnehin dünnen Personaldecke, die bei der Arbeitsverwaltung Brandenburgs bestehe, die Schließung aller Nebenstellen drohe, wenn die Einsparung der Personalkosten um wei-

46 Ebenda, Staatssekretär Peschke am 12. 7. 1950 an den ZKSK-Vorsitzenden Fritz Lange.

47 Vgl. dazu: Braun, Die Zentrale Kommission für Staatliche Kontrolle; Klawitter, Die Rolle der ZKK; Kos, Politische Justiz in der DDR.

t8 BAB, DC 1/1536, Fritz Lange am 14.7. 1950 an das ZS [sic] der SED (Max Herm).

49 BAB, DQ 2/906, Bl. 144-150, Hauptabteilungsleiter Litke am 12. 7. 1950 an den stellvertretenden Ministerpräsidenten Walter Ulbricht. Ein gleichlautendes Schreiben mit anderem Datum (18.7.) bcfindet sich in: BAB, DC $1 / 1536$.

50 BAB, DQ 2/906, Bl. 144-150, hier Bl. 145, Litke am 12. 7. 1950 an Ulbricht.

$51 \mathrm{Vgl}$. Hoffmann, Sozialpolitische Neuordnung, S. 291. 
tere 10 Prozent 1950 wiederholt werde ${ }^{52}$. Die einzelnen Arbeitsämter seien gegenüber den anderen Ländern stark unterbesetzt und es lägen bereits „zahlreiche Einsendungen und Beschwerden der Bevölkerung und der Massenorganisationen" vor, die diesen Zustand kritisieren würden. Grobbel bat daher um eine Befreiung seiner Verwaltung von der Kürzungsauflage. Das Ministerium für Arbeit und Gesundheitswesen schloß sich zunächst der Argumentation des Landesamtes in Potsdam an und erwog sogar für Brandenburg eine Erhöhung der Planstellenzahlen ${ }^{53}$. Zum Ausgleich sollten bei den übrigen Ländern entsprechend mehr Stellen gekürzt werden. Nach ersten Gesprächen mit den Ministerien der Finanzen sowie des Innern, die an der Sparaktion beteiligt waren, entband Minister Steidle das brandenburgische Landesamt von der Pflicht, im laufenden Jahr weitere Personalkürzungen durchzuführen ${ }^{54}$. Er stellte im Zusammenhang mit der Erstellung eines neuen Strukturplanes für die Arbeitsverwaltung eine Erhöhung der Stellenzahlen in Aussicht. Obwohl das Arbeitsministerium in Berlin seine Zusicherung gegeben hatte, lag eine entsprechende Anweisung des Finanzministeriums auch Ende August noch nicht vor. So sah sich der brandenburgischen Finanzminister gezwungen, auf der Umsetzung der im Haushaltsplan vorgesehenen Kürzungen zu bestehen ${ }^{55}$. Bei dieser Auseinandersetzung zwischen der Arbeits- und Finanzverwaltung konnte sich letztere durchsetzen. Minister Steidle mußte den Antrag Brandenburgs, von der angeordneten Personaleinsparung um 10 Prozent befreit zu werden, ablehnen und die in Aussicht gestellte Aufstockung des Verwaltungspersonals einstweilen zurückziehen ${ }^{56}$. Gleichzeitig wies Steidle das Landesamt an, vorsorglich ausgesprochene Kündigungen bei den Arbeitsschutzinspektoren, die von zentraler Bedeutung für die Durchführung des Gesetzes der Arbeit seien, "sofort" rückgängig zu machen. Von den Sparmaßnahmen sollte „in erster Linie das Personal für ausgesprochene Verwaltungsaufgaben betroffen werden“; konkretere Vorschläge vermied allerdings der Minister. Die brandenburgische Landesregierung folgte der Anweisung aus Berlin und löste das Finanzproblem selber, indem bisher gesperrte Haushaltsmittel freigegeben wurden ${ }^{57}$.

Bei den Angestellten der Arbeitsämter verbreitete sich im Sommer 1950 zunehmende Unsicherheit, die mit der eben beschriebenen ungewissen Finanzlage zusammenhing. Hinzu kamen vereinzelte, zum Teil gewaltsame Interventionen von seiten der Volkspolizei, die zu einer Verschlechterung der Stimmungslage auch in der Bevölkerung beitrugen. Das mecklenburgische Ministerium für Arbeit und Gesundheitswesen berichtete über eine Polizeiaktion am 13. Juni bei sämtlichen Arbeitsämtern sowie den Neben- und Hilfsstellen des Landes, in deren Verlauf die bei den Ämtern geführten Karteikarten, soweit sie Angehörige der Volkspolizei betrafen, sichergestellt wurden. Minister Werner Pöhls (CDU) beklagte sich daraufhin über die Durchführung der Aktion: „Die ganze Angelegenheit hätte

52 BAB, DQ 2/903, Bl. 43, Minister Grobbel am 4. 5. 1950 an den Minister für Arbeit und Gesundheitswesen Steidle.

53 Ebenda, Bl. 42, Aktennotiz Schulz vom 13. 5. 1950.

54 Ebenda, Bl. 40, Minister Steidle am 19.5. 1950 an Minister Grobbel.

55 Dies geht aus einem gemeinsamen Schreiben des Innen- und Arbeitsministeriums Brandenburgs vom 22. 8. 1950 hervor. Ebenda, Bl. $36 \mathrm{f}$.

56 Ebenda, Bl. 33 f., Minister Steidle am 31. 8. 1950 an Minister Grobbel.

57 Ebenda, Bl. 32, Minister Grobbel am 14. 9. 1950 an Minister Steidle. 
sich völlig reibungslos abwickeln lassen, wenn der Chef der Volkspolizei mich [Minister Pöhls] vorher unterrichtet hätte und wenn z.B. durch eine gemeinsame Anordnung die Herausgabe der Karteikarten an die Volkspolizei geregelt worden wäre." 58 Der Minister für Arbeit und Gesundheitswesen Mecklenburgs beabsichtigte sogar, den Ministerrat in die Angelegenheit einzuschalten und richtete an Minister Steidle die Bitte, beim Minister des Innern vorstellig zu werden, „damit die Arbeit im Geschäftsbereich meines Ministeriums nicht durch derartige Polizeiaktionen lahm gelegt wird"59. Reaktionen des Innenministeriums sowie des Ministerrates blieben jedoch aus bzw. liegen nicht vor. Festzuhalten bleibt die wachsende Unsicherheit auf seiten der Arbeitsverwaltung und indirekt wohl auch bei der Bevölkerung - darauf weisen einige Berichte der Arbeitsämter bzw. der Landesämter hin. Vor diesem Hintergrund wurde die Diskussion über eine Neuordnung der Arbeitsverwaltung im Herbst 1950 fortgesetzt.

Ende September befaßte sich das Sekretariat des ZK der SED mit der angestrebten Neustrukturierung der Arbeitsverwaltung und bestätigte im wesentlichen die von Litke vorgelegten Grundprinzipien ${ }^{60}$. Kleine Abweichungen traten nur bei den Zahlenangaben auf: So ging das SED-Führungsgremium von insgesamt 127 Kreisarbeitsämtern aus, die „auf etwa 50 “ zu reduzieren waren. Das Ministerium für Planung erhielt den Auftrag, zusammen mit dem Ministerium für Arbeit und Gesundheitswesen die Planungsarbeiten fortzusetzen. In dem Zusammenhang spielten für die SED-Führung aber nicht das Ziel einer Zusammenlegung einzelner Kreise die entscheidende Rolle, sondern vielmehr die bevorzugte Versorgung der wirtschaftlichen Großprojekte des Fünfjahrplanes. Die verbleibenden $50 \mathrm{Ar}-$ beitsämter sollten nämlich ,in den Schwerpunkten unserer industriellen Entwicklung für die Gegenwart und für die Zukunft gesehen liegen“. Dabei stellten die politischen Grenzen der Kreise und Länder nach den Vorstellungen des Sekretariats kein Hindernis dar. Gleichzeitig wurden die Aufgaben der Arbeitsämter grob skizziert, die den engen Zusammenhang von Verwaltungsreform und inhaltlicher Ausrichtung der Arbeitskräftelenkung deutlich werden ließen: $\mathrm{Zu}$ den zentralen Aufgaben gehörten „Werbung" und Vermittlung von Arbeitskräften „für die volkswirtschaftlich wichtigsten Objekte im Fünfjahrplan"61. Des weiteren sollten alle „verfügbaren“ Arbeitskräfte, insbesondere Facharbeiter statistisch erfaßt und für die ökonomischen Großvorhaben mobilisiert werden. Die Arbeitsämter hatten sich außerdem verstärkt der Frauenerwerbstätigkeit sowie der beruflichen Eingliederung von Schulabgängern zuzuwenden. Dieser Aufgabenkatalog enthielt keine grundsätzlichen Neuerungen. Bemerkenswert an ihm war jedoch zum einen die Verknüpfung mit der Verwaltungsreform und zum anderen die Einbettung in den ersten Fünfjahrplan. Abschließend sprach sich das Sekretariat des ZK für eine „Regelung der Arbeitsverhältnisse in den Privatbetrieben“62 aus, ohne jedoch weiter konkret zu werden.

58 Ebenda, Bl. 13f., hier Bl. 13, Vermerk Pöhls vom 14. 6. 1950.

59 Ebenda, Bl. 12, Minister Pöhls am 15.6. 1950 an Minister Steidle.

60 SAPMO, DY 30/J IV 2/3/141, Bl. 11 f., Protokoll der Sitzung des Sekretariats des ZK am 28. 9. 1950.

61 Ebenda.

62 Ebenda, Bl. 12. 
Das Ministerium für Arbeit und Gesundheitswesen war offensichtlich vorab über die Entscheidung der SED-Führung informiert worden, so daß noch am selben Tag ein neuer Verordnungsentwurf ausgearbeitet werden konnte, der sich sehr eng am Beschluß des Sekretariats des ZK orientierte ${ }^{63}$. Bei einer erneuten Überarbeitung wurde die zentrale Position, welche das Ministerium bei der Arbeitskräftelenkung ohnehin einnahm, wieder besonders hervorgehoben ${ }^{64}$. Ende des Monats lag eine ausführlich gehaltene Durchführungsbestimmung vor, die nunmehr auch die inhaltlichen Fragen der Arbeitskräftelenkung regeln sollte65. Diese faßte unter anderem die geltenden Bestimmungen zur Registrierung der Bevölkerung kurz zusammen und schrieb die Aufgaben und Kompetenzen der Arbeitsämter nochmals fest. Dabei wurden die Arbeitsämter in ihrer Funktion etwas aufgewertet: Sie sollten erneut als Schaltstellen der Arbeitskräftelenkung fungieren. In dem Zusammenhang hatte nicht nur jeder Betrieb seinen Bedarf ausschließlich dort zu melden; auch die betrieblichen Einstellungen und Entlassungen bedurften der Zustimmung durch das zuständige Amt für Arbeit ${ }^{66}$. Darüber hinaus mußten Inserate vor der Veröffentlichung genehmigt werden. Die Bestimmungen zu den Instrumenten der Arbeitsverwaltung für die Lenkung und Steuerung des Arbeitskräftepotentials bedeuteten einen gewissen Rückschritt. Im Vordergrund hatte zwar nach wie vor die Werbung zu stehen; in Ausnahmefällen konnte aber auf die Arbeitseinweisung zurückgegriffen werden. Das galt zum einen bei der Beseitigung von „öffentlichen Notständen“ sowie der Erfüllung von "Produktionsprogrammen in den Wirtschaftsschwerpunkten" ${ }^{67}$. Damit verblieb dem jeweiligen Arbeitsamt ein nicht unbeträchtlicher Interpretationsspielraum. Insgesamt gesehen stellte die geplante Durchführungsbestimmung nur die $\mathrm{Zu}$ sammenfassung anderer arbeitsrechtlicher Verordnungen, Anweisungen und sowjetischer Befehle aus der Zeit zwischen 1945 und 1948 dar, die damit aufgehoben werden sollten 68 .

Als das Sekretariat des ZK der SED am 27. November 1950 den Verordnungsentwurf über die Reorganisation der Ämter für Arbeit „als Grundlage“ annahmm ${ }^{69}$, schien sich eine baldige Veröffentlichung und damit ein rasches Inkraftreten abzuzeichnen. Auf Wunsch der SED-Führung sollten nur noch zwei Überarbeitungsvorschläge in den Entwurf einfließen. Zum einen war ein zusätzlicher Paragraph einzufügen, „in dem die Aufgaben der Arbeitsämter präzisiert werden“. Da dies in der bereits erstellten Durchführungsbestimmung geschehen war, blieb die Anweisung des Sekretariats etwas unklar. Möglicherweise war bereits zu diesem Zeitpunkt eine Zusammenlegung der beiden Gesetzestexte zu einer Gesamtverordnung geplant. Zum zweiten wurde die Zusammenlegung einzelner Arbeitsäm-

$63 \mathrm{BAB}, \mathrm{DQ} 2$ 2/1757, Entwurf der Abt. Arbeitskraftlenkung vom 28. 9. 1950.

64 Ebenda, 2. Verordnungsentwurf der Abt. Arbeitskraftlenkung vom 2. 11. 1950. Dieser Entwurf wurde anschließend mit dem Ministerium für Planung abgestimmt und ging wenige Tage später an das ZK der SED. Vgl. BAB, DE 1/11742, Bl. 1-4, Bruno Leuschner am 8. 11. 1950 an das ZK der SED (Scholz).

65 $\mathrm{BAB}, \mathrm{DQ} 2 / 1729$, Entwurf des Ministeriums für Arbeit und Gesundheitswesen (Abt. III) vom 24. 11.1950.

66 Ebenda, S. 5 ( $\$ 9)$.

67 Ebenda $(\$ 10)$.

68 Der Entwurf listete 19 gesetzliche Bestimmungen auf. Vgl. ebenda, S. 10-12 (\$24).

69 SAPMO, DY 30/J IV 2/3/156, Bl. 17. 
ter in Sachsen, Sachsen-Anhalt und Thüringen angeregt. Sowohl die Landesverwaltungen als auch das Ministerium für Arbeit und Gesundheitswesen lehnten die von der ZK-Abteilung Wirtschaftspolitik vorgeschlagene Zusammenlegung einzelner Arbeitsämter $\mathrm{ab}^{70}$.

Anfang 1951 wurden erstmals Stimmen laut, die eine Auflösung der Arbeitsämter verlangten. Für einen solchen Schritt warb etwa der stellvertretende Ministerpräsident und Vorsitzende der Staatlichen Plankommission (SPK), Heinrich Rau, der als Begründung die Beseitigung der Arbeitslosigkeit angab. Die ursprünglich zentrale Aufgabe der Arbeitsverwaltung, Arbeitslose zu betreuen, sei somit entfallen. Die übrigen arbeitsmarktpolitischen Aufgaben könnten zudem von anderen staatlichen Verwaltungen übernommen werden. So erfordere etwa die Lehrlingsausbildung sowie die berufliche Unterbringung von Jugendlichen „nicht den Apparat der Arbeitsämter, da diese Arbeit in erster Linie in Verbindung mit den Gewerkschaften und den Fachministerien bzw. Vereinigungen [der Volkseigenen Betriebe] organisiert und durchgeführt werden muß" " Rau verband diese Einschätzung mit einer deutlichen Kritik an der bisherigen Tätigkeit der Arbeitsämter: „Die Lenkung der Arbeitskräfte, die mehr und mehr in einer Umsetzung von Betrieb zu Betrieb besteht, wurde von den Arbeitsämtern bisher in so geringem Maße durchgeführt, daß schon daraus sich zeigt, daß sie dazu nicht in der Lage sind. "72 Auch diese Aufgabe solle in Zukunft von den Fachministerien in Zusammenarbeit mit den VVB und den Gewerkschaften gelöst werden. Rau schlug vor, eine Kommission des Arbeitsministeriums und der Gewerkschaften in die Sowjetunion und in andere osteuropäische Staaten zu entsenden ${ }^{73}$, um die „bestehende Organisation auf diesem Gebiet [zu] studieren und dann die notwendigen Maßnahmen vor[zu]schlagen“. Abschließend stellte Heinrich Rau - und das war höchst aufschlußreich - seine Forderung nach Auflösung der Arbeitsämter in einen gesamtdeutschen Kontext, der die aus Sicht der SED- und DDR-Führung zu Beginn der fünfziger Jahre immer stärker einsetzende Systemauseinandersetzung verdeutlichte. Angesichts der zu diesem Zeitpunkt in der Bundesrepublik steigenden Erwerbslosenzahlen könne die DDR die Vorzüge des eigenen Wirtschafts- und Gesellschaftssystems propagandistisch unterstreichen: „Die Tatsache, daß in Westdeutschland die Arbeitslosigkeit steigt, könnte gegenüber gestellt werden der Tatsache der Liquidierung der Arbeitslosigkeit bei uns, der Auflösung der Arbeitsämter und daß das Bestehen der volkseigenen Betriebe eine unbürokratische Lenkung der Arbeitskräfte ermöglicht.“

Das Ministerium für Arbeit, das noch am 9. Januar einen umfassenden Perspektivplan für die einzelnen Hauptabteilungen verfaßt hatte, in dem die arbeitsmarktpolitischen Aufgaben einen herausgehobenen Platz einnahmen ${ }^{74}$, widersprach den Überlegungen des stellvertretenden Ministerpräsidenten. Arbeitsminister Chwalek betonte einleitend zu seinem ausführlichen Antwortschreiben: „Von vornher-

$70 \mathrm{BAB}, \mathrm{DQ} 2 / 493$, Hausmitteilung an Minister Chwalek vom 15. 12. 1950.

71 BAB, DQ 2/1713, Rau am 6.1. 1951 an Arbeitsminister Chwalek, S. 1.

72 Ebenda, S. 2.

73 Vgl. zu den Delegationsreisen als Möglichkeit sowjetischer Einflußnahme die kurzen Überlegungen von Kaiser, Sowjetischer Einfluß, S. 121-123.

74 BAB, DE 1/11277, Bl. 7-11, Perspektivplan des Ministeriums für Arbeit (Sekretariat Minister Chwalek) vom 9.1. 1951. 
ein kann ich Dir mitteilen, daß sich unsere Gedanken geradezu kreuzen." 75 Chwalek hielt zwar das langfristige Ziel, die Arbeitsämter aufzulösen und deren Aufgaben auf die Fachministerien zu verteilen, für diskussionswürdig. Zuvor müßten allerdings eine ganze Reihe von Voraussetzungen geschaffen werden: Dazu zählten die verstärkte Initiative von seiten der Gewerkschaften sowie der Aufbau einer neuen Abteilung Arbeitskraft in den jeweiligen Ministerien: „Wir müssen dazu gelangen, daß sie [die Fachministerien] sich alle eine Abt. Arbeitskraft schaffen, die sich mit den Fragen der Wettbewerbs- und Aktivistenbewegung, der Lohnpolitik, der Sozial- und Kulturpolitik in den Betrieben, der Arbeitskraftlenkung, der Normenerstellung und insbesondere des Arbeitsschutzes befassen. Haben wir diese Voraussetzungen, so können wir allmählich an den Abbau der Ämter für Arbeit und darüber hinaus unter Berücksichtigung der gesamtpolitischen Situation auch an die Auflösung des gesamten Ministeriums für Arbeit denken. "76 Der Arbeitsminister verhielt sich in dieser Angelegenheit erstaunlich passiv und plädierte sogar dafür, zunächst die noch ausstehende Entscheidung im Sekretariat des ZK abzuwarten. Das SED-Führungsgremium hatte am 11. Januar einer inhaltlichen Erweiterung der in Planung befindlichen Verordnung zugestimmt: Diese führte nochmals alle Tätigkeitsbereiche der Arbeitsämter auf, verpflichtete die Arbeitsämter zur Zusammenarbeit mit den Betrieben, Industriegewerkschaften und gesellschaftlichen Organisationen und legte die Zusammenlegung von zwei Ämtern (Magdeburg und Burg) fest 7 .

Bei der Auseinandersetzung über eine mögliche Auflösung der Arbeitsämter besaß die Staatliche Plankommission die besseren Karten, da die SKK offensichtlich hinter diesem Vorhaben stand. Darüber hinaus besaß das Ministerium für Arbeit allem Anschein nach keinen vergleichbar engen Kontakt zur sowjetischen $\mathrm{Be}$ satzungsmacht wie die von Heinrich Rau geführte zentrale Planungsbehörde ${ }^{78}$. Der Leiter der Abteilung für Wirtschaftsplanung, W. A. Chomjakow, berichtete dem stellvertretenden SKK-Vorsitzenden G. S. Bukow am 17. Januar 1951 ausführlich über die geplante Reorganisation der ostdeutschen Arbeitsverwaltung. Dabei gelangte er zu der Schlußfolgerung, daß die von Arbeitsminister Chwalek eingeleiteten „Maßnahmen halbherzig sind und nicht in vollem Maße die mit der Versorgung der Wirtschaft der Republik mit Arbeitskräften zusammenhängenden Fragen lösen, sowie die unnötige Registrierung von ,Arbeitslosen' nicht beseitigen"79. Im einzelnen kritisierte Chomjakow die Vielzahl arbeitsrechtlicher Bestimmungen, die jedoch teilweise auf sowjetische Befehle oder Anweisungen zurückzuführen waren, die bei den Arbeitsämtern zu einer „bürokratischen Anwendung“ sowie zu einem "rein formale[n] Arbeitsstil“ geführt hätten ${ }^{80}$. Be-

75 BAB, DQ 2/1713, Chwalek am 15.1. 1951 an Rau, S. 1.

76 Ebenda, S. 4.

77 SAPMO, DY 30/J IV 2/3/166, Bl. $5 \mathrm{f}$.

78 Laut SKK-Statut war die sowjetische Abt. für Wirtschaftsplanung unmittelbar zuständig für die Kontrolle des Ministeriums für Planung. Vgl. SKK-Statut, S. 123.

79 AVP RF, f. 458, op. 72, p. 258, d. 14, l. 58. Ich danke meiner Arbeitskollegin, Frau Dr. Elke Scherstjanoi, daß sie mir dieses Dokument zur Verfügung gestellt hat. Die Ubersetzung fertigte Frau Katrin Reichelt an, der ich ebenfalls herzlich danken möchte.

80 AVP RF, f. 458, op. 72, p. 258, d. 14, 11. 59-64, hier 1. 61, Mitteilung der SKK-Abt. für Wirtschaftsplanung über die bestehende Praxis der Registrierung der nichtarbeitenden Bevölkerung durch die Abteilungen für Arbeit/Arbeitsämter. 
anstandung fand vor allem die Arbeitslosenstatistik, die dazu beitrage, "daß die Erfassung der Arbeitslosen in den Abteilungen für Arbeit zur einfachen Formalität und offensichtlich irreführend und verfälschend geworden ist". Der SKK-Mitarbeiter sprach sich damit indirekt für eine Bereinigung und Vereinfachung der Statistiken aus, um die Zahl der Erwerbslosen drastisch zu verringern. Den Arbeitsämtern wurde vorgehalten, sie kämen aufgrund der „übertriebene[n] und platzraubende[n] Erfassung " nicht mehr zu ihrer eigentlichen Aufgabe: der Versorgung der einzelnen Wirtschaftsbereiche mit Arbeitskräften. Darüber hinaus übte Chomjakow Kritik an der gesetzlichen Beschränkung von Arbeitseinweisungen, in erster Linie durch die Verordnung vom 2. Juni 1948: „Dieser Beschluß beinhaltet so viele Vorbehalte bezüglich des Arbeitseinsatzes, daß jeder, der nicht zu arbeiten wünscht, die Möglichkeit hat, nicht zur Arbeit zu gehen." ${ }^{81}$ Das bedeutete nicht automatisch eine Rückkehr zu den "harten“ Maßnahmen der Arbeitsverpflichtung vor 1948/49. Die sowjetische Militäradministration hatte selber den qualitativen Wechsel der Arbeitsmarktpolitik hin zur Anwerbung unterstützt. Vielmehr machten die Ausführungen Chomjakows das Unverständnis auf seiten der SKK deutlich, daß sich die deutsche Arbeitsverwaltung eigene Beschränkungen auferlegt hatte, die zudem von jedem einzelnen Arbeiter zum Teil eingeklagt werden konnten. Die Möglichkeit, im Bedarfsfall auf die Methode der Arbeitsverpflichtung zurückgreifen zu können, sollte letztlich gewahrt und nicht zu sehr eingeengt werden. Die vom Leiter der SKK-Unterabteilung Arbeit und Löhne P. Masanow eingebrachten Vorschläge verfolgten zwei Ziele: Zum einen ging es darum, die mit der statistischen Erhebung beauftragten Stellen davon abzubringen, die gesamte Bevölkerung zu erfassen. Zum zweiten sollten die Fachministerien bei der Einstellung von Arbeitskräften sowie deren zwischenbetrieblicher Steuerung beteiligt werden ${ }^{82}$. Die weitere Diskussion innerhalb der SKK kann aufgrund des Quellenmangels nicht weiter rekonstruiert werden. Es kann aber festgehalten werden, daß die für wirtschafts- und arbeitsmarktpolitische Fragen zuständige Abteilung eindeutig Stellung nahm zugunsten der SPK, und somit gegen das Ministerium für Arbeit. Nachdem Chomjakow seinen Standpunkt am 6. Februar gegenüber Chwalek erläutert hatte, zeigte sich dieser plötzlich sehr kompromißbereit ${ }^{83}$ : Seine zunächst vorgebrachten Einwände bestanden nun nicht mehr.

Anfang 1951 verdichteten sich die Hinweise auf eine baldige Auflösung der Arbeitsämter in ihrer bestehenden Form. Dazu trugen nicht nur die geschilderten Vorstöße der SPK und die positive Stellungnahme der SKK bei, sondern auch die bereits angekündigten Kontakte zu anderen ostmitteleuropäischen Staaten. So hatte etwa die Staatssekretärin im Ministerium für Arbeit, Friedel Malter, eine Unterredung mit ihrem tschechoslowakischen Amtskollegen geführt. Demzufolge existierten dort keine Arbeitsämter ${ }^{84}$ : Die Aufgabe der Arbeitskräftewerbung übernahmen vielmehr die Komitees der Nationalen Front und die Ortsgruppen des Demokratischen Frauenbundes, die im übrigen ehrenamtlich arbeite-

81 Ebenda, 1. 62 .

82 Ebenda, 1.63.

$83 \mathrm{BAB}, \mathrm{DQ}$ 2/1713, Chwalek am 7. 2. 1951 an das ZK der SED (Stoph).

${ }_{84} \mathrm{BAB}, \mathrm{DQ}$ 2/717, Aktennotiz Malters vom 5. 2. 1951. 
ten. Zur Mobilisierung neuer Arbeitskräfte wurden alle Fachministerien eingeschaltet. Malter sprach sich dafür aus, den DFD für die arbeitsmarktpolitischen Aufgaben in der DDR stärker einzubinden, und griff eine Anregung von Käthe Kern auf, die sich allerdings vergeblich darum bemüht hatte, die Frauenorganisation in die Werbung weiblicher Arbeitskräfte einzuschalten. Diese Form der Mitarbeit könne jedoch - so Malter - nicht für die Nationale Front gelten, die einen „etwas anders gearteten Charakter" habe.

Das Ministerium für Arbeit paßte sich der neuen Entwicklung erstaunlich schnell an und legte bereits am 17. Februar 1951 einen Verordnungsentwurf vor, der die Übertragung der Arbeitskräftelenkung auf die Fachministerien vorsah ${ }^{85}$. Die Arbeitsämter fanden dabei keine Erwähnung mehr; ihre Aufgabengebiete sollten anderen Verwaltungen zugewiesen werden. So war die Abteilung Arbeitsschutz den Wirtschaftsministerien der Länder anzugliedern; die Aufgaben der Abteilung Arbeitsproduktivität erhielten die Fachministerien. Der Verordnungsentwurf sah außerdem vor, daß das Zentralamt für Statistik die statistischen Aufgaben übernehmen sollte, wobei die Einwohnerkartei der Städte und Gemeinden "entsprechend den Erfordernissen der Arbeitskraftlenkung umzugestalten“ war ${ }^{86}$. Berufsberatung und berufliche Betreuung der Jugendlichen fielen wiederum dem Staatssekretariat für Berufsausbildung zu. Zur Absicherung einer bedarfsgerechten Lenkung sah der Entwurf die Bildung sogenannter Arbeitskraftlenkungsausschüsse auf zentraler Ebene, Landes- und Kommunalebene vor, die sich aus Vertretern des Arbeitsministeriums, der Fachministerien, Gewerkschaften und Massenorganisationen zusammensetzen sollten.

Das Ministerium für Arbeit griff die sich ausweitende Kritik an der Tätigkeit der Arbeitsverwaltung auf und zählte die einzelnen Punkte, die einer reibungslosen Lenkung angeblich im Wege standen, durchaus selbstkritisch auf. Dabei paßten sich die leitenden Mitarbeiter der stark ideologisch aufgeladenen Methode an, "Kritik und Selbstkritik“ zu üben. So räumte beispielsweise Hauptabteilungsleiter Kreil in einer hausinternen Denkschrift ein: „Trotz der Entwicklung unserer Wirtschaftsplanung hinderten Unkenntnis der sowjetischen Methoden bezüglich der Lenkung der Arbeitskraftreserven sowie mangelhafte marxistisch-leninistische Erkenntnis in der Weiterentwicklung unserer gesamten Volkswirtschaft die Angestellten der Ämter für Arbeit, in genügendem Maße zu erkennen, daß die Zeit der Vermittlungen von Arbeitskräften vorbei ist und eine tatsächliche Lenkung derselben durchgeführt werden muß." ${ }^{87}$ Dahinter stand vermutlich auch der Versuch einer weitgehenden Bestandssicherung: So hatte sich zwar Minister Chwalek gegenüber den Vorschlägen zur Auflösung der Arbeitsämter gesprächsbereit gezeigt, innerhalb des Ministeriums machte er jedoch keinen Hehl daraus, daß er einen solchen Schritt grundsätzlich ablehnte ${ }^{88}$. Seiner Ansicht nach war ohne Arbeitsämter den nach wie vor nicht unbeträchtlichen Anforderungen von

85 BAB, DQ 2/1729, Entwurf der Abt. III. Der Ministerrat hatte auf seiner Sitzung am 15. 2. 1951 einen entsprechenden Auftrag an das Arbeitsministerium beschlossen. Vgl. BAB, DQ 2/493, Chef der Regierungskanzlei am 19.2. 1951 an den Minister für Arbeit.

86 BAB, DQ 2/1729, Entwurf der Abt. III, S. 3.

87 BAB, DQ 2/1734, Exposé vom 19. 2. 1951, S. 2.

$88 \mathrm{BAB}, \mathrm{DQ}$ 2/493, Protokoll der Arbeitstagung des Ministeriums für Arbeit am 20.2. 1951, S. 1. 
seiten der sowjetischen Besatzungsmacht, Arbeitskräfte für den Uranbergbau bereitzustellen, nicht nachzukommen. Chwalek sprach sich dafür aus, nicht so sehr die organisatorischen Fragen in den Vordergrund zu stellen, sondern vielmehr das Problem zu diskutieren, „wie die Arbeit in der Zukunft verbessert und operativer gestaltet werden kann". Darüber hinaus mußte das Ministerium für Arbeit einräumen, daß die Arbeitsämter die ihnen auferlegten Erwartungen bisher nicht hatten erfüllen können. Dieses Eingeständnis bezog sich zum einen auf das grundlegende Problem der nicht steuerbaren Fluktuation sowie die Tatsache, daß ein Großteil der Stellenvermittlung an den Arbeitsämtern vorbeilief. So meldete etwa das Amt für Arbeit in Dresden, daß rund 60 Prozent der gemeldeten offenen Stellen durch Eigeninitiative der Arbeitsuchenden abgedeckt worden seien ${ }^{89}$.

Das Ministerium für Arbeit favorisierte auch in der Folgezeit die Verringerung der Anzahl der Kreisarbeitsämter von 127 auf nunmehr $55^{90}$ und versuchte dadurch, die Arbeitsverwaltung als eigene Verwaltungseinheit zu erhalten. Gleichzeitig machte das Ministerium Zugeständnisse gegenüber seinen Kritikern, die auf eine Übertragung der Aufgaben auf die Fachministerien drängten. Demzufolge sollte bei der Arbeitskräftezuteilung eine Aufgabenteilung entsprechend der Wirtschaftsform vorgenommen werden: Die Abteilungen für Arbeit in den Stadtund Landkreisen sollten ,ihre Haupttätigkeit zukünftig im privatwirtschaftlichen Teil unserer Volkswirtschaft finden ", während die Fachministerien auch in diesem Bereich für die volkseigene und ihnen gleichgeschaltete Industrie letztlich verantwortlich sein sollten ${ }^{91}$. Das lief auf einen Kompetenzverlust sowie eine Arbeitsentlastung hinaus, da sich die bisher bestehende Konfliktebene zwischen Arbeitsverwaltung und Betrieben stärker zu den Fachministerien verlagert hätte. Ein ähnliches Ziel verfolgte auch der im Arbeitsministerium einmütig unterbreitete Vorschlag, das Arbeitsbuch nicht mehr weiter zu nutzen und die entsprechenden Verordnungen außer Kraft zu setzen ${ }^{92}$. Dieses Instrument hatte vor allem in der Frühphase zum schlechten Ansehen der Arbeitsämter unter der Erwerbsbevölkerung beigetragen. Vertreter des Arbeitsministeriums sowie der Industrieministerien ${ }^{33}$ einigten sich am 22. März darauf, die existierenden Ämter für Arbeit aufzulösen und zugleich Abteilungen für Arbeit bei den Stadt- und Landkreisen zu errichten ${ }^{94}$. Erstmals wurden auch die Betriebe in die Arbeitskräftelenkung aktiv eingebunden: Sie sollten im volkseigenen Sektor zuständig sein für die Anwerbung der benötigten Arbeiter 95 . Andererseits wurde das Ausmaß der Fluktuation noch stärker als bisher an das Verhalten der einzelnen Betriebsleitungen gekoppelt, d.h. die betriebliche Sozialpolitik gewann an Bedeutung: „Betriebe, die die sozialen Belange der Belegschaft nicht wahren, müssen eben mit einer Abkehr der

89 BAB, DQ 2/1734, Exposé Kreils vom 19. 2. 1951, S. 3.

$90 \mathrm{BAB}, \mathrm{DQ} 2 / 1713$, Vorschläge Peschkes für die Reorganisation des Ministeriums für Arbeit und Gesundheitswesen (o.D.), S. 2.

$91 \mathrm{BAB}, \mathrm{DQ} 2 / 493$, Niederschrift vom 22.3. 1951 über die Verhandlungen der Kommission zur Frage der Reorganisation der Ämter für Arbeit, S. 1.

92 Ebenda, S. $1 \mathrm{f}$.

93 Ministerium für Maschinenbau, Hauptverwaltung Kohle, Ministerium für Schwerindustrie, Ministerium für Leichtindustrie. Darüber hinaus waren noch zwei Mitarbeiter der Landesregierung Sachsen-Anhalt anwesend.

94 BAB, DQ 2/493, Niederschrift über die Sitzung am 22.3. 1951.

95 Ebenda, S. 4. 
Arbeiter rechnen. "96 Materielle Anreize sollten auf diese Weise für einen Arbeitsplatzwechsel an Bedeutung gewinnen - eine Strategie, die bereits mit dem SMADBefehl Nr. 234 angelegt worden war. Die Veränderung bestand nunmehr aber darin, daß die Betriebe in den Planungen der staatlichen Verwaltungen sehr viel stärker als handelnde Akteure berücksichtigt wurden. Auch hierbei befand sich die DDR-Regierung jedoch rasch in einem Dilemma: Auf der einen Seite ging es um die Eindämmung der Fluktuation, auf der anderen Seite sollten die Betriebe ihren Arbeitskräftebedarf selbst abdecken. Dafür nahmen die Vertreter des Arbeitsministeriums und der Industrieministerien ein Ansteigen der Fluktuationsrate zunächst in Kauf. Diese würde - so die Überlegung - langfristig wieder sinken.

Die Übereinkunft, die zwischen dem Arbeitsministerium und den Industrieministerien gefunden werden konnte, fand schnell Eingang in die weiteren Planungsarbeiten. Daran zeigte sich, daß das Ministerium für Arbeit sehr stark in die Defensive geraten war und die Initiative mittlerweile vollständig verloren hatte. Ende März hatte Hauptabteilungsleiter Kreil sein Exposé für eine Neuordnung der Arbeitsämter erneut umgearbeitet und dabei vor allem den Beschluß über die Aufgabentrennung zwischen Arbeitsverwaltung und Fachministerien eingearbeitet ${ }^{97}$. In diesem Zusammenhang übernahm er erstmals die Einschätzung, die bisher nur von den übrigen Ministerien vertreten worden war, wonach die Arbeitsämter in ihrer bestehenden Form nicht in der Lage seien, den Anforderungen des Fünfjahrplanes gerecht zu werden. Verhandlungen mit Vertretern der Planökonomischen Abteilung der SKK zeigten, daß die sowjetische Besatzungsmacht mit der Ausarbeitung der geplanten Verordnung weitgehend einverstanden war ${ }^{98}$. Ihre Kritik bezog sich in erster Linie auf formale Fragen.

Obwohl innerhalb des Ministeriums für Arbeit ursprünglich Einigkeit darüber bestanden hatte, das Arbeitsbuch abzuschaffen, meldeten sich Ende April 1951 einige Befürworter zu Wort, darunter die hausinterne Abteilung Arbeitsrecht. Diese vertrat den Standpunkt, daß es "außerordentlich zweckmäßig“ gewesen sei, "den Beschäftigten das Arbeitsbuch und damit jederzeit die Möglichkeit zu geben, nachzuweisen, welche berufliche Qualifikation er hat und welche Arbeitsplätze er inne hatte" 99 . Des weiteren wurde kritisiert, daß die Sozialämter entsprechend dem Verordnungsentwurf die Organisation der Arbeitslosenversicherung zu übernehmen hatten. Darin erblickte die Abteilung eine Rückkehr „zu einer Fürsorgeunterstützung alten Stils“. Dagegen versuchte Minister Chwalek die Ausarbeitung der Verordnung grundsätzlich zu verzögern und hinauszuschieben: Man dürfe nicht von einer Reorganisation oder gar Auflösung der Arbeitsämter ausgehen, sondern vielmehr von der „Neuregelung der Arbeitskraftlenkung und den sonst noch der Arbeitsverwaltung verbleibenden Aufgaben" ${ }^{100}$. Mit dem vor-

${ }^{96}$ Ebenda, S. 3.

$97 \mathrm{BAB}, \mathrm{DQ} 2$ 2/1734, Exposé Kreils vom 27. 3. 1951. Eine erweiterte Fassung vom 28. 3. 1951 findet sich in: $B A B, D Q 2 / 493$.

${ }_{98} \mathrm{BAB}, \mathrm{DQ} 2 / 493$, Bericht der Abt. Arbeitskraftlenkung über eine Besprechung bei der SKK am 6. 4. 1951 .

99 Ebenda, Hausmitteilung an das Sekretariat des Ministers vom 26. 4. 1951.

100 Ebenda, persönlicher Referent des Ministers Giersch am 4. 5. 1951 an Abt. Arbeitskraftlenkung (Heisig). 
liegenden Verordnungsentwurf war Chwalek überhaupt nicht einverstanden: „So wie die Dinge im Augenblick im 5. Entwurf ventiliert sind, ist die Frage nicht zu lösen."

Die Abteilung Arbeitskraftlenkung setzte sich über einige hausinterne Veränderungsvorschläge hinweg. So sah der 6. Entwurf vom 5. Mai 1951, der die Überschrift „Neugestaltung der Arbeitskraftlenkung“ trug, eine Eingliederung der Ämter für Arbeit in die Kreisverwaltungen vor, welche die Lenkung der in der privaten Wirtschaft Beschäftigten durchzuführen hatten ${ }^{101}$. Die neu zu gründenden Abteilungen Arbeitskraft bei den Fachministerien erhielten die Zuständigkeit für die innerbetriebliche und überbetriebliche Arbeitskraftlenkung, und zwar für die volkseigene Industrie. Diese Aufteilung entsprechend der Wirtschaftsform war somit erhalten geblieben, hatte jedoch nicht die vollständige Unterstützung durch den Arbeitsminister erhalten. Eine Rückkehr zum Prinzip der Arbeitseinweisung war ausgeschlossen, da die sogenannten Umsetzungen von Arbeitskräften nur mit Zustimmung der davon betroffenen Arbeitskräfte vorgenommen werden konnten. Zahlreiche Aufgaben wurden trotz der erhobenen Einwände auf die Sozialämter übertragen: die Erfassung von Schwerbeschädigten sowie die Betreuung der pflichtversicherten Arbeitslosen. Diese Bestimmung stand allerdings dem langfristigen Ziel einer raschen Ausdünnung der Sozialfürsorge mit entsprechenden Einsparungen bei den Personalkosten im Wege. Während die Verordnung über die Pflichtversicherung gegen Arbeitslosigkeit ausdrücklich aufgehoben wurde, enthielt der Entwurf keine Angaben zur Arbeitsbuchverordnung, die offenbar noch weiter Bestand hatte.

Minister Chwalek blieb bei seiner ablehnenden Haltung, wies allerdings gleichzeitig die zuständige Abteilung an, „die Arbeiten so zu beschleunigen, daß die ganze Verordnung innerhalb [von] 14 Tagen vorlagereif“ sei102. Das kam dem Eingeständnis einer Niederlage gleich, da sich der Arbeitsminister mit seiner grundlegenden Kritik am Verordnungsentwurf nicht hatte durchsetzen können. Seinen Widerstand gab er nunmehr auf und ließ die Abteilung Arbeitskraftlenkung bei der Fertigstellung der gesetzlichen Neuregelung gewähren. Er mahnte nur noch dazu, beim Aufbau der Abteilungen für Arbeit in den Kreisverwaltungen „nicht schematisch [zu] verfahren" 103 . Seine Anregung bezog sich auf die personelle $\mathrm{Be}-$ setzung der Abteilungen, die flexibel gehandhabt werden müsse. Um die inhaltliche Abstimmung mit den übrigen Ministerien möglichst rasch abzuschließen, lud das Ministerium für Arbeit am 10. Mai zu einer Arbeitstagung ein ${ }^{104}$. Dort wurden grundsätzliche Einwände gegen die Neuordnung der Verwaltung sowie die Neuverteilung der Zuständigkeitsgebiete nicht mehr vorgetragen. Die einge-

$101 \mathrm{BAB}, \mathrm{DQ} 2 / 1757$.

$102 \mathrm{BAB}, \mathrm{DQ} 2 / 493$, Minister Chwalek am 9. 5. 1951 an Abteilungsleiter Heisig, S. 1.

103 Ebenda, S. 2.

$104 \mathrm{BAB}, \mathrm{DQ} 2 / 1204$, Protokoll über die Arbeitstagung am 10. 5. 1951. Anwesend waren Vertreter aus folgenden Ministerien, Verwaltungen bzw. Massenorganisationen: Ministerium für Post- und Fernmeldewesen, Ministerium für Leichtindustrie, Staatliche Plankommission, Ministerium der Finanzen, Ministerium für Maschinenbau, Ministerium des Innern, Ministerium für Handel und Versorgung, Ministerium für Land- und Forstwirtschaft, Ministerium für Verkehr, FDGB-Bundesvorstand, Magistrat von Groß-Berlin, Generaldirektion Schiffahrt sowie Generaldirektion Kraftverkehr und Straßenwesen. 
brachten Änderungswünsche betrafen offensichtlich nur die Gliederung der Verordnung sowie stilistische Fragen. Eine inhaltliche Präzisierung wurde dagegen bei der Festlegung der betrieblichen Aufgabengebiete vorgenommen: Es herrschte bei den Beteiligten Konsens darüber, daß „es nicht Aufgabe der Arbeitskraftlenkung in den Betrieben sein kann, die Arbeitskräftepläne selbst zu erarbeiten, sondern daß dieses Aufgabe der Planabteilung" sei ${ }^{105}$. Zu den offengebliebenen Fragen gehörte vor allem die Registrierung bzw. statistische Erfassung der erwerbsfähigen Bevölkerung; auch die Weiterführung der soeben aufgebauten betrieblichen Arbeitskräftekartei war noch zu klären. Hier mußten noch mit der Staatlichen Plankommission und dem Ministerium des Innern Verhandlungen geführt werden ${ }^{106}$.

Gegen die in der Verordnung enthaltene strikte Trennung zwischen volkseigener und privater Wirtschaft wurden frühzeitig Bedenken vorgebracht. So hielt beispielsweise der Magistrat von Groß-Berlin eine Aufteilung der Arbeitskräftekartei für nicht sehr praktikabel, da die nach wie vor bestehende Fluktuation von Arbeitskräften zwischen beiden Wirtschaftsformen „einen vermehrten Verwaltungsaufwand durch das Hin- und Hersenden der Karteikarten erforderlich machen" würde ${ }^{107}$. Die Magistratsverwaltung verlangte eine Sonderregelung für Berlin, da hier die Fluktuation besonders stark verbreitet sei. Auch auf seiten der betroffenen Arbeitsämter, die sich bisher Zurückhaltung auferlegt hatten, entwikkelte sich vorsichtiger Widerspruch gegen die geplante Auflösung bzw. Eingliederung in die Kommunalverwaltung: Die Arbeitsamtsleiter in Sachsen-Anhalt verabschiedeten am 18. Mai 1951 eine Entschließung, in der sie die allgemein beschworene Verbesserung der Verwaltungsarbeit durch das Reformvorhaben in Frage stellten. Die Einbettung in die Kreisverwaltung bedeute „keine Verkürzung des Arbeitsganges, sondern eine Verlängerung". Die erhoffte Personaleinsparung würde - so die Leiter der Arbeitsämter - nicht eintreten, „sondern im Gegenteil eine stärkere Belastung der für die Verwaltung zuständigen Arbeitskollegen" ${ }^{108 .}$ Zahlreiche Mitarbeiter der Arbeitsverwaltung befürchteten offensichtlich einen Bedeutungsverlust, der sich etwa in der Ausstattung mit Personal- und Sachmitteln niederschlagen werde. Auch die zukünftige Zusammenarbeit innerhalb der Kreisverwaltungen wurde skeptisch beurteilt: Der Leiter des Arbeitsamtes in Riesa machte darauf aufmerksam, „daß er in seiner langen Praxis bei der Stadtoder Kreisverwaltung für die Belange des Arbeitsamtes bisher noch nie Verständnis gefunden hätte “109. Um die Eigenständigkeit der Arbeitsverwaltung auch weiterhin zu erhalten, schlug das Arbeitsamt in Herzberg/Elster vor, die neu geschaffenen Abteilungen als selbständige Dezernate innerhalb der Kreisverwaltung zu

105 Ebenda, S. 3.

$106 \mathrm{BAB}, \mathrm{DQ} 2 / 1165$, Disposition des Ministeriums für Arbeit (Abt. Arbeitskraftlenkung) vom 12. 5. 1951, S. 3 .

107 BAB, DQ 2/1713, Magistrat von Groß-Berlin (Abt. Arbeit und Gesundheitswesen) am 15. 5. 1951 an das Ministerium für Arbeit (Abt. Arbeitskraftlenkung).

108 BAB, DQ 2/1538.

109 Ebenda, Bericht des Ministeriums für Arbeit (Abt. III) über die Dienstreise nach Riesa am 17./18. 5. 1951, S. 1 . 
etablieren ${ }^{110}$. Auf diese Weise bleibe die rasche und zentrale Durchführung der Volkswirtschaftspläne und damit auch der Arbeitskräftepläne gewahrt.

Obwohl Arbeitsminister Roman Chwalek seinen grundsätzlichen Widerstand aufgegeben hatte, versuchte er dennoch in der Folgezeit, Verbesserungsvorschläge einzubringen, die jedoch so allgemein gehalten waren, daß die bei der Ausarbeitung der Verordnung beteiligten Ressorts darauf nicht einzugehen brauchten. $\mathrm{Da}$ bei ließ Chwalek unverhohlen erkennen, daß er Zweifel an der fachlichen Kompetenz der anderen Ministerien hatte, was seine mittlerweile isolierte Position innerhalb der DDR-Regierung verstärkt haben dürfte. So betonte er etwa gegenüber Innenminister Karl Steinhoff, die geplante Eingliederung der Arbeitsämter in die Kreisverwaltungen sei „eine Verwaltungsangelegenheit von so bedeutendem Ausmaß“, daß mit der Fertigstellung der Verordnung ein Mitarbeiter beauftragt werden solle, „der uns wirklich eine Hilfe sein kann"111. Gegenüber der ZKAbteilung Wirtschaft deutete Chwalek eine mögliche ablehnende Haltung der sowjetischen Besatzungsmacht zu den Neuordnungsplänen an. Diese Einschätzung, die mit der bisher geschilderten Position der SKK unvereinbar war, speiste sich aus einer Unterredung, welche zwei leitende Mitarbeiter des Arbeitsministeriums mit „den Freunden“ geführt hatten. Die SKK-Vertreter waren angeblich der Meinung, daß auf die Ämter für Arbeit „als Kontrollorgan“ prinzipiell nicht verzichtet werden könnte ${ }^{12}$. Im Gegensatz dazu hatte die planökonomische Abteilung der SKK die bestehende Struktur der Arbeitsverwaltung stark in Frage gestellt. Darüber hinaus werde das Staatssekretariat für Berufsausbildung Einspruch gegen Teile der Verordung erheben und dabei die Zuständigkeit bei der Berufsausbildung für sich einfordern. Chwalek erklärte, daß es vorerst nicht möglich sei, die Arbeitskräftelenkung dort anzusiedeln, da arbeitsökonomische Gründe dagegen sprächen: „Wenn das Staatssekretariat für Berufsausbildung es in der nächsten Zeit fertig bringt, sich um die Berufsausbildung zu kümmern [...], dann haben sie eine solche gewaltige Aufgabe, die sie nicht einmal verdauen können."113

Bei einzelnen Bestimmungen des Verordnungsentwurfes bestanden Ende Mai 1951 immer noch Unstimmigkeiten zwischen dem Arbeitsministerium und der SKK. So vertrat der sowjetische Vertreter Nassanow die Meinung, daß es nicht möglich sei, die Arbeitsbuchkartei in ihrer jetzigen Form weiterbestehen zu lassen, da zeitgleich eine drastische Reduzierung der Personal- und Sachkosten angestrebt werde ${ }^{14}$. Dagegen betonte der Leiter der Abteilung Arbeitskraftlenkung Heisig, daß diese Kartei „das einzige Instrument ist, welches Aufschluß darüber gibt, wo wer arbeitet" 115 . Unterschiedliche Ansichten existierten außerdem bei der Frage, wer zukünftig für die Erstellung der statistischen Erhebungen verantwortlich sein sollte. Nassanow erklärte in dem Zusammenhang, daß dies „nicht Aufgabe der Abteilungen für Arbeit sein könne, sondern vielmehr Sache der der

$110 \mathrm{BAB}, \mathrm{DQ} 2$ 2/1713, Amt für Arbeit und Sozialfürsorge Herzberg/Elster am 23.5. 1951 an das Ministerium für Arbeit.

111 BAB, DQ 2/493, Chwalek am 18. 5. 1951 an Steinhoff.

112 Ebenda, Chwalek am 19. 5. 1951 an die ZK-Abt. Wirtschaft der SED (Scholz), S. 1.

113 Ebenda, S. 2.

$114 \mathrm{BAB}, \mathrm{DQ} 2 / 1713$, Aktenvermerk über die am 23.5. 1951 bei der SKK Karlshorst stattgefundene Besprechung, S. 1 .

115 Ebenda, S. 2. 
Staatlichen Plankommission nachgeordneten Organe". Dadurch ließen sich zudem weitere Verwaltungskosten einsparen. Abteilungsleiter Heisig entgegnete auf diesen Vorschlag mit dem Hinweis, daß die von den Arbeitsämtern angefertigte Statistik „die einzige brauchbare Quelle ist, welche Aufschluß gibt über die Arbeitskräftezahlen nach regionalen Gesichtspunkten". Die Staatliche Plankommission benötige dieses Zahlenmaterial im übrigen - so Heisig weiter - „dringend“ zur Vorbereitung des Volkswirtschaftsplanes 1952. Die Einwände der SKK waren allerdings nicht grundsätzlicher Natur: Einen Tag später lenkte der Mitarbeiter der SKK ein und beschränkte sich auf auf einige unbedeutende Anregungen zum Verordnungsentwurf ${ }^{116}$. Er ließ sich außerdem von den beiden Vertretern des Arbeitsministeriums, Heisig und Krüger, davon überzeugen, daß die Verwaltungsreform nicht die von der SKK erhofften Einsparungsmöglichkeiten bieten werde. Heisig ging aufgrund der vorgesehenen Errichtung entsprechender Abteilungen in den Fachministerien, VVB und Betrieben sogar von einem Ansteigen der Personalkosten aus ${ }^{117}$.

Eine nicht unwesentliche Veränderung ergab sich dadurch, daß Aufgaben der Arbeitslosenversicherung nicht mehr - wie ursprünglich vorgesehen - den Sozialämtern, sondern der Sozialversicherung zugeordnet werden sollten. Der vom Arbeitsministerium ausgearbeitete Entwurf vom 25. Mai118 paßte sich insofern der übergeordneten sozialpolitischen Diskussion an, die nunmehr ein "Absterben“ der Sozialfürsorge und - langfristig gesehen - eine Übernahme der Sozialversicherung durch den FDGB vorsah. Nachdem das Ministerium für Arbeit mit dem Ministerium des Innern die Endredaktion vorgenommen hatte, wandte sich Chwalek an den stellvertretenden Ministerpräsidenten Rau mit der Bitte, letzte Änderungsvorschläge vorzubringen ${ }^{119}$. Anschließend sollte der Verordnungsentwurf dem Sekretariat des ZK der SED zur Beschlußfassung vorgelegt werden, das auf der Sitzung am 21. Juni 1951 seine grundsätzliche Zustimmung gab ${ }^{120}$. Bestätigung fand des weiteren ein vorgelegter Entwurf für die 1. Durchführungsbestimmung. Eine endgültige Verabschiedung war aber erst für Ende Juni geplant, sobald die endgültige Formulierung über die Struktur der neu zu schaffenden Ämter vorlag. Arbeitsminister Roman Chwalek und der Staatssekretär im Finanzministerium Willi Georgino schlugen vor, die Arbeitsämter bis zum 31. August 1951 aufzulösen und deren Aufgaben auf die Kreisverwaltungen zu übertragen ${ }^{121}$.

Obwohl der endgültige Entwurf bereits vorlag, der im Laufe des Monats Juni die grundsätzliche Zustimmung der betroffenen Ministerien und des FDGB gefunden hatte ${ }^{122}$, dauerten die abschließenden redaktionellen Arbeiten noch etwas an. Der SPK-Vorsitzende Rau regte einige Verbesserungen bzw. Klarstellungen im Verordnungsentwurf an, die sich auf die Abgrenzung der Aufgabengebiete zwischen den Kreisverwaltungen und den Fachministerien bei der Erstellung der Ar-

$116 \mathrm{BAB}, \mathrm{DQ} 2 / 1713$, Aktenvermerk über die Besprechung am 24. 5. 1951, S. 3.

117 Ebenda, S. 2.

118 BAB, DQ 2/1204.

119 BAB, DQ 2/493, Chwalek am 12.6. 1951 an Rau.

120 SAPMO, DY 30/J IV 2/3/206, Bl. 2.

121 BAB, DC 20 Teilbestand Ulbricht/3960, Bl. 2, Entwurf für eine Ministerratsvorlage (o.D.).

$122 \mathrm{BAB}, \mathrm{DQ} 2 / 493$, Aktennotiz der Abt. Arbeitskraftlenkung vom 26. 6. 1951. 
beitskräftepläne sowie der Berufsausbildung beschränkten ${ }^{123}$. Bereits einen Tag später stimmte das Politbüro der Verordnung sowie der Durchführungsbestimmung „als Grundlage“ zu, wobei allerdings eine erneute „Schlußredaktion“ vorgenommen werden sollte ${ }^{124}$. Diese Aufgabe sollten Walter Ulbricht und Heinrich Rau übernehmen. Gleichzeitig erteilte die SED-Führung Roman Chwalek den Auftrag, zusammen mit dem Amt für Information und dem FDGB-Bundesvorstand eine öffentlichkeitswirksame Kampagne vorzubereiten, bei der besonders der Zusammenhang mit dem Fünfjahrplan deutlich gemacht werden sollte. Die Verabschiedung der Verordnung durch den Ministerrat verzögerte sich jedoch Ende Juni, weil nun die sowjetischen „Freunde“ neue Einwände vorgebracht hatten ${ }^{125}$. Diese lassen sich aber anhand der deutschen Aktenüberlieferung inhaltlich nicht näher bestimmen. Das Politbüro verabschiedete auf seiner Sitzung am 10. Juli endgültig die beiden Entwürfe ${ }^{126}$. Arbeitsminister Chwalek erhielt den Auftrag, die Verordnung ,in der Regierung einzubringen.“ Dort wurde die „Verordnung über die Aufgaben der Arbeitsverwaltungen und über die Lenkung der Arbeitskräfte" am 12. Juli beschlossen ${ }^{127}$ und konnte somit in Kraft treten ${ }^{128}$. An die Stelle einer Durchführungsbestimmung trat zunächst ein sehr kurz gehaltener Durchführungsbeschluß129.

Aufgrund dieser Verordnung konnten die Kommunalverwaltungen der Stadtund Landkreise eigene Abteilungen für Arbeit errichten, welche die Aufgaben der Arbeitsämter zu übernehmen hatten. Der dreigliedrige Aufbau der Arbeitsverwaltung bestand damit nicht mehr: Die untere Stufe war weggebrochen. Auf den ersten Blick war das Rad der Geschichte zurückgedreht worden - auf die Zentralisierung folgte nun die Kommunalisierung. Die lang andauernde Debatte in den ersten Nachkriegsjahren über das Ordnungsprinzip für diese Verwaltung hatte bekanntlich die starke Vereinheitlichung und Zentralisierung zum Ergebnis gehabt. Die Verordnung vom 12. Juli 1951 schien nunmehr den Kritikern von einst recht zu geben und hätte als Stärkung der kommunalen Körperschaften angesehen werden können. Dieser Eindruck täuscht allerdings darüber hinweg, daß die neu zu schaffenden Abteilungen für Arbeit auch weiterhin der Anleitung und Anweisung durch die Hauptabteilungen für Arbeit des zuständigen Fachministeriums der Landesregierungen bzw. durch das Ministerium für Arbeit in Berlin unterworfen blieben. Die neu konzipierte Verwaltungsstruktur war eben primär den Erfordernissen der zentralen Wirtschaftsplanung geschuldet und sollte die Fachministerien mit einbeziehen. Mit diesem Schritt bewegte sich die DDR letztlich auf das sowjetische Vorbild stärker zu: In der Sowjetunion nahmen die Industrieministerien unter anderem die Aufgaben der Arbeitsverwaltung wahr, die als eigener Verwaltungsstrang nicht existent war. Auf der anderen Seite bedeutete die Auflösung der Arbeitsämter einen Kontinuitätsbruch in der deutschen Geschichte. Die DDR verabschiedete sich von Strukturmerkmalen des Verwaltungs-

${ }^{123}$ BAB, DE 1/11277, Bl. 15, Rau am 25. 6. 1951 an Arbeitsminister Chwalek.

124 SAPMO, DY 30/IV 2/2/154, Bl. 19, Protokoll der Sitzung des Politbüros am 26. 6. 1951.

125 BAB, DQ 2/493, Staatssekretär Malter am 30.6. 1951 an Minister Chwalek.

126 SAPMO, DY 30/IV 2/2/156, Bl. 85.

$127 \mathrm{BAB}, \mathrm{DC} 20 \mathrm{I} / 3-59, \mathrm{Bl} .6$ und 38-43.

128 Gesetzblatt der DDR 1951, S. 687-689.

129 BAB, DC 20 I/3-59, Bl. 44. 
aufbaus, die in der Weimarer Republik angelegt worden waren und nach $1945 \mathrm{zu}$ nächst aufgegriffen und fortgeführt wurden. Daß dieser Bruch aber nicht vollständig war, beweist die Tatsache, daß die Kreisverwaltungen in Zukunft die Aufgaben der Arbeitsämter übernehmen sollten. Die Verordnung vom 12. Juli 1951 machte darüber hinaus deutlich, daß die SED-Führung nach der nahezu vollständigen Beseitigung der Arbeitslosigkeit in eine neue Phase eintreten wollte, in der die Aufgaben der bedarfsgerechten Arbeitskräfteplanung und -zuteilung im Mittelpunkt zu stehen hatten. Die vom Zusammenbruch und der Bewältigung der unmittelbaren Kriegsfolgen geprägte erste Periode sollte demonstrativ abgeschlossen werden. Die Planungsexperten in der SED-Führung und der SPK waren offensichtlich davon überzeugt, daß sie sich nunmehr den eigentlichen Aufgaben einer sozialistischen Wirtschaftspolitik zuwenden konnten.

Die Verordnung war jedoch nicht nur ein Instrument zur Stärkung der Zentralverwaltungswirtschaft auf dem Gebiet der Arbeitskräfteallokation, sondern bot auch den Betrieben und den Arbeitern neue Handlungsspielräume. Während die Betriebe die Erlaubnis erhielten, ihren Arbeitskräftebedarf durch eigene Werbung selber abzudecken, wurde zugleich den einzelnen Arbeitern indirekt die Möglichkeit eingeräumt, den Arbeitsplatz zu wechseln. Beides war zuvor von den Arbeitsämtern stark eingeschränkt worden. Damit deutete sich aber ein neuer Zielkonflikt mit der Reduzierung der Fluktuationsrate an. Da jedoch die Arbeitsämter dieses Problem nicht hatten lösen können, schien das Risiko aus Sicht der SED-Führung sowie der DDR-Regierung nicht allzu groß gewesen zu sein.

Als nächstes mußte die geplante Durchführungsbestimmung zur Regelung einiger offener Detailfragen auf den Weg gebracht werden. Dazu hatte das Arbeitsministerium bereits wenige Tage nach dem Inkrafttreten der Verordnung einen ersten Entwurf ausgearbeitet ${ }^{130}$. Von zentraler Bedeutung waren dabei die Bestimmungen zur Registrierung der erwerbsfähigen Bevölkerung sowie die Erfassung der sogenannten Arbeitskraftreserve. Darunter fielen Arbeitsuchende und Jugendliche, die nicht vom Nachwuchsplan erfaßt wurden, sowie Sozialfürsorgeempfänger und Schwerbeschädigte. Der Minister für Schwerindustrie Fritz Selbmann wollte dem vorgelegten Entwurf seine Zustimmung nicht geben: Er entspreche „in einigen Teilen [nicht] dem Sinn der Verordnung “131. Nach Ansicht Selbmanns befanden sich die Bestimmungen zu den Registrier- und Kontrollkarten im Widerspruch zum Verordnungstext vom 12. Juli. Der Hauptkritikpunkt des Ministers für Schwerindustrie richtete sich jedoch gegen die Beteiligung der Arbeitsverwaltung bei der Anwerbung von Arbeitskräften für die vom Ministerrat festgelegten Schwerpunktbetriebe. Um eine „schnelle Lösung der Aufgaben der Arbeitskräftewerbung " zu garantieren, sollten die Landesministerien für Arbeit sowie die neuen Abteilungen für Arbeit nur beratend hinzugezogen werden, so die Forderung Selbmanns. Arbeitsminister Chwalek wies die vorgetragene Kritik zum Teil zurück und bemühte sich, die Zustimmung Selbmanns zum ausgearbeiteten Entwurf zu gewinnen. Im einzelnen machte er darauf aufmerksam, daß sowohl die Registrier- als auch die Kontrollkarte „unerläßlich“ für die Arbeits- 
kräfteplanung und -lenkung seien ${ }^{132}$. Auf beides könne nicht verzichtet werden. Zugleich unterstrich Chwalek, daß das Arbeitsministerium eine eher koordinierende Funktion wahrnehmen werde, und bestätigte explizit die erweiterten $\mathrm{Zu}$ ständigkeiten der Fachministerien. Es sei Aufgabe der einzelnen Ministerien, Staatssekretariate sowie der volkseigenen Betriebe, „in den Schwerpunktbetrieben bzw. volkswirtschaftlich bedeutungsvollen Objekten in erster Linie selbst für die Abdeckung des bestehenden Arbeitskräftebedarfs Sorge zu tragen"133. Eine Ausnahme machte dabei aber nach wie vor der Erzbergbau in Sachsen und Thüringen. Aufgrund des hohen Arbeitskräftebedarfs für den Uranbergbau sah sich das Arbeitsministerium gezwungen, direkte Auflagen gegenüber einzelnen Betrieben zu erheben. Auch der Minister für Maschinenbau Gerhart Ziller äußerte Kritik am Entwurf des Arbeitsministeriums und beanstandete vor allem, daß die Durchführungsbestimmung ,in ihrer Gesamtheit [...] überhaupt zu viel auf die Belange der Länderebene abgestellt" sei134. Diese Kritik wies der Arbeitsminister als unbegründet zurück und verwies darauf, daß die „Arbeitskräftepläne der Industrieministerien [...] die notwendige Berücksichtigung in der Durchführungsbestimmung gefunden" hätten ${ }^{135}$. Gleichzeitig bat er Ziller „dringend“ um Zustimmung zum vorgelegten Entwurf. Nachdem die Unstimmigkeiten zwischen dem Arbeitsministerium und den beiden genannten Industrieministerien - von den übrigen lagen keine Einwände vor - ausgeräumt werden konnten, trat die 1. Durchführungsbestimmung am 7. August 1951 in $\mathrm{Kraft}^{136}$.

Es ist bereits darauf hingewiesen worden, daß die Arbeitskräftesteuerung mit Hilfe dieser Verordnung immer stärker in die Planwirtschaft eingebettet wurde. Das Arbeitsministerium verlor nicht nur durch die Auflösung der Arbeitsämter an Bedeutung, sondern auch durch die Kompetenzerweiterung anderer Ministerien. Die Fachministerien waren nunmehr primär zuständig für die Bedarfsabdekkung in ihrem Zuständigkeitsbereich. Darüber hinaus wurde den Betrieben - auch das ist bereits mehrfach betont worden - eine aktive Rolle zugewiesen ${ }^{137}$. Eine koordinierende Funktion besaß neben dem Arbeitsministerium die Staatliche Plankommission, die zunehmend an Bedeutung gewinnen sollte138. Aufgrund dieser Vergrößerung der Anzahl der beteiligten Akteure schien die Befürchtung einer Kompetenzüberschneidung und damit einer Verlangsamung der Entscheidungsprozesse durchaus naheliegend zu sein. Für die Wirtschaftsplaner innerhalb der SED-Führung sowie der DDR-Regierung stellte sich jedoch diese Problematik

132 BAB, DQ 2/493, Chwalek am 28. 7. 1951 an Selbmann, S. 1.

133 Ebenda, S. 2.

134 BAB, DQ 2/1714, Ziller am 31. 7. 1951 an Chwalek.

$135 \mathrm{BAB}, \mathrm{DQ}$ 2/493, Chwalek am 7.8.1951 an Ziller.

136 Gesetzblatt der DDR 1951, S. 753-755.

137 Das Ministerium für Arbeit (Abt. Planung, Investitionen, Statistik) versandte im Oktober 1951 ein entsprechendes Merkblatt an die Betriebe. BAB, DQ 2/1204. Zur Werbung von Arbeitskräften waren nicht nur die volkseigenen Betriebe, sondern auch die Privatbetriebe berechtigt. BAB, DQ 2/2126, Abt. Arbeitskraftlenkung am 19. 11. 1951 an die Vereinigte Holzstoff- und Papierfabriken A.G. in Niederschlemma.

${ }^{138}$ Hinzu kamen die noch bestehenden Landesverwaltungen, die zwar weisungsabhängig waren, gleichzeitig aber noch über einen gewissen Handlungsspielraum verfügten. Vgl. LA Magdeburg LHA, Rep. K MW, Nr. 9448, Bl. 75, Ministerium für Arbeit (Litke) am 27. 8. 1951 an das Ministerium für Wirtschaft (HA Arbeit) in Sachsen-Anhalt. 
nicht. Im Gegenteil: Alle Beteiligten hatten offenbar die Gewißheit, auf diese Weise die Zuweisung des Produktionsfaktors ,Arbeit' insgesamt verbessert zu haben.

Der erhoffte Erfolg bei der Personaleinsparung trat teilweise ein. Nach Berechnungen des Ministeriums für Arbeit lag die prozentuale Reduzierung der Arbeitsstellen in den einzelnen Ländern zwischen 25,9 (Sachsen) und 34,1 (Thüringen) ${ }^{139}$. Damit hatten die SPK und das Arbeitsministerium zumindest ein Nahziel erreicht. Die eigentliche Bewährungsprobe stand den Abteilungen für Arbeit aber noch bevor: Die Planung und Lenkung der Arbeitskräfte in Zusammenarbeit mit den Fachministerien und den Betrieben. Die Aufteilung der Zuständigkeiten auf die neu geschaffenen Abteilungen in den Kreisverwaltungen (Privatindustrie) sowie die Fachministerien (volkseigene Industrie) erleichterte dieses Unterfangen nicht sonderlich. Das langfristige Ziel war nicht nur die bedarfsorientierte Arbeitskräftelenkung, sondern auch die Reduzierung der Fluktuationsrate. Hier mußte sich zeigen, ob die neue Verwaltungsstruktur endlich Abhilfe schaffen konnte.

\section{Gesetz über den Mutter- und Kinderschutz und die Rechte der Frau}

Das Gesetz der Arbeit hatte das Arbeitsministerium zusammen mit den Fachministerien und Landesverwaltungen aufgefordert, Maßnahmen zu ergreifen, um die Gruppe der nicht erwerbstätigen Frauen für die „Schwerpunkte der Wirtschaft“ zu gewinnen. Darüber hinaus verpflichtete das Gesetz alle Betriebe und Verwaltungen unverbindlich, „in weitestem Umfang Arbeitsplätze mit weiblichen Arbeitskräften zu besetzen " 140 . Zugleich erhielt das Arbeitsministerium den Auftrag, Durchführungsbestimmungen auszuarbeiten, welche die berufliche Eingliederung von Frauen zu erleichtern hatten: Dazu zählte vor allem der Auf- und Ausbau von Kindergärten "und anderen sozialen Einrichtungen" 141 , durch die die Frauen bei ihrer familiären und häuslichen Arbeit erheblich entlastet werden sollten. Im folgenden Abschnitt soll der Frage nachgegangen werden, wie sich die Zielsetzung der Gewinnung weiblicher Arbeitskräfte im Arbeitsrecht der frühen DDR niederschlug.

Bereits vor der DDR-Gründung gab es Pläne, ein Gesetz zu verabschieden, das nicht nur die Einbindung von arbeitsfähigen Frauen in den Produktionsprozeß, sondern auch staatliche Hilfen für Mütter und Kinder vorsah sowie allgemeine Bestimmungen zur Rolle der Frau in Familie und Gesellschaft enthalten sollte. So lag etwa dem Zentralsekretariat der SED ein Gesetzentwurf „zum Schutze der Mütter und Kinder" vor, der jedoch nach kurzer Diskussion und ohne Angabe von Gründen zunächst zurückgestellt wurde ${ }^{142}$. Erst Anfang 1950 wurde dieses Vorhaben wieder aufgegriffen: Am 7. Februar legte Käthe Kern von der Abteilung Mutter und Kind beim Ministerium für Arbeit und Gesundheitswesen ${ }^{143}$ einen

139 BAB, DQ 2/493, Bericht des Ministeriums für Arbeit vom 5. 9. 1951, S. 3.

140 Gesetzblatt der DDR 1950, S. 349-355, hier S. 352 (\$27, Abs. 1).

141 Ebenda ( $\$ 27$, Abs. 2).

142 SAPMO, DY 30/IV 2/2.1/246, Protokoll der Sitzung des Zentralsekretariats vom 1. 11. 1948.

${ }^{143}$ Die Kompetenzen der Abteilungen Arbeitskraftlenkung sowie Mutter und Kind waren klar von- 
Entwurf für ein „Mutterschutzgesetz“ vor, der sich primär mit Fragen des Arbeits- und Kündigungsschutzes von werdenden Müttern beschäftigte sowie finanzielle Hilfen von seiten der Sozialversicherung, der staatlichen Verwaltungen und Betriebe auflistete ${ }^{144}$. In ihrer Begründung zum Gesetzentwurf wies Kern darauf hin, daß das von den nationalsozialistischen Machthabern 1942 erlassene Mutterschutzgesetz noch nicht außer Kraft gesetzt worden sei und vor allem nicht mehr den sozialpolitischen Zielen der DDR entspreche, „nämlich die Arbeitskraft zu erhalten und sinnvoll anzusetzen [sic]“145.

Mittlerweile hatte jedoch der Demokratische Frauenbund Deutschlands (DFD) unter der Leitung von Elli Schmidt die Federführung bei der weiteren Ausarbeitung des Gesetzes erhalten; das Arbeitsministerium sollte dagegen nur noch beratend mitwirken können ${ }^{146}$. Dahinter standen vermutlich propagandistische Motive: Der DFD sollte dem Ministerpräsidenten bei einem offiziellen Empfang am 7. März - und damit rechtzeitig zum Internationalen Frauentag am 8. März einen entsprechenden Vorschlag unterbreiten und damit die Eigenständigkeit der Massenorganisation demonstrieren ${ }^{147}$. Der Entwurf der Frauenorganisation betonte sehr viel stärker als der von Käthe Kern das vorrangige Ziel, Frauen verstärkt in die Arbeitswelt einzubeziehen. So wurde einleitend die Bedeutung der Volkswirtschaftspläne für den weiteren Aufbau der DDR unterstrichen und auf den steigenden Arbeitskräftebedarf hingewiesen. Dem vom Arbeitsministerium sowie der SED-Führung eingebrachten Lösungsansatz, nicht erwerbstätige Frauen für den Produktionsprozeß zu gewinnen, schloß sich der DFD vorbehaltlos an und betonte: „Der notwendige Mehrbedarf an Arbeitskräften wird in erster Linie von Frauen bestritten werden müssen." Daran knüpfte sich die Forderung an, Maßnahmen einzuleiten, „die es den Frauen ermöglichen, mehr als bisher beruflich tätig zu sein" ${ }^{148}$. Der DFD wollte sich zugleich für die Besetzung von qualifizierten Arbeitsplätzen mit Frauen einsetzen und damit einen Austausch von männlichen durch weibliche Hilfsarbeiter unterbinden: „Das alte Vorurteil, daß Frauen für qualifizierte Berufe [...] nicht geeignet sei[e]n, muß überwunden werden. Sie sollen nicht nur als Hilfsarbeiterinnen Beschäftigung finden, sondern als qualifizierte, den Männern in der Leistung gleichwertige Arbeitskräfte ausgebildet werden." ${ }_{149}$ Dieses ambitionierte Ziel tangierte zu einem wesentlichen Teil die Berufsausbildung; detaillierte Vorstellungen besaß die Frauenorganisation dazu aber nicht. Der Entwurf enthielt außerdem Vorschläge, die auf eine partielle Aushöhlung des bestehenden Arbeitsschutzes für berufstätige Frauen hinausliefen. So wurde das Ministerium für Arbeit und Gesundheitswesen aufgefordert, gemein-

einander abgegrenzt. Der Abt. Mutter und Kind blieb es überlassen, „ideologische Vorbereitungsarbeiten zu leisten und eine bessere soziale Betreuung der arbeitenden Frau im Betrieb anzustreben". Für die Lenkung weiblicher Arbeitskräfte war dagegen nach wie vor die Abt. Arbeitskraftlenkung zuständig. BAB, DQ 2/1726, Abteilungsleiter Krüger am 21.2. 1950 an das Ministerium für Arbeit und Gesundheitswesen von Sachsen. Diese Aufgabenabgrenzung galt demzufolge auch für die Landesverwaltungen.

144 SAPMO, NY 4090/563, Bl. 6-11.

145 Ebenda, Bl. 3-5, hier Bl. 3, Begründung Kerns vom 23. 2. 1950.

146 Ebenda, Bl. 1, Notiz (o.Verf.) vom 20. 2. 1950.

147 SAPMO, NY 4090/563, Bl. 27-37, Vorschläge des DFD zur Förderung der Frauen vom 7. 3. 1950.

148 Ebenda, Bl. 29.

149 Ebenda, Bl. 31. 
sam mit dem FDGB zu überprüfen, „wo Arbeiten oder Berufe für Frauen verboten sind, ob und welche Möglichkeiten bestehen, solche Verbote aufzuheben oder solche technischen Veränderungen zu schaffen, die es den Frauen ermöglichen, solche Arbeit auszuführen" 150 .

Die Frauenabteilung beim SED-Parteivorstand unterbreitete dem Politbüro am 17. April 1950 einen eigenen Entwurf für ein „Gesetz zur Förderung der Frau“151, den das SED-Führungsgremium einen Tag später annahmm ${ }^{152}$. Da dieser jedoch sehr kurz gehalten war, verabschiedete das Politbüro zugleich einen ausführlicher gehaltenen Gesetzentwurf ${ }^{153}$. In der Folgezeit mußte eine Absprache mit dem Justizministerium erzielt werden, das für die Erstellung des familienrechtlichen Teils des geplanten Gesetzes verantwortlich war ${ }^{154}$. Da an dem Verfahren auch noch die Regierungskanzlei beteiligt war, die in Zusammenarbeit mit dem Ministerium für Volksbildung einen allgemeinen Teil über die "gesellschaftliche Stellung der Frau“ zu verfassen hatte ${ }^{155}$, verzögerte sich die weitere Ausarbeitung zusätzlich. Ungeklärt blieb die von Staatssekretär Peschke aufgeworfene Frage nach der Finanzierung des im Gesetz vorgesehenen Ausbaus von Kindergärten ${ }^{156}$. Dabei mußte entschieden werden, ob die anfallenden Kosten der Sozialversicherung oder den Kommunalverwaltungen aufgebürdet werden sollten. Am 20. Mai lag erstmals ein vollständiger Gesetzentwurf vor, der die geplanten Abschnitte zusammenfaßte, die zuvor von den beteiligten Ressorts erstellt worden waren ${ }^{157}$. Kernstück des Gesetzes bildete der vom Arbeitsministerium erarbeitete Abschnitt, der detailliert auf Maßnahmen zur beruflichen Förderung von Frauen in den drei Wirtschaftssektoren (Landwirtschaft, Industrie, öffentliche Verwaltung) einzugehen versuchte. Bei der SKK fand der vorgelegte Entwurf allerdings keine Zustimmung: Sie kritisierte, daß das Gesetz eine „Deklaration“ sei und keine konkreten Bestimmungen enthalte ${ }^{158}$. Gleichzeitig konnte Grotewohl, der offensichtlich ein $\mathrm{Ge}-$ spräch mit sowjetischen Vertretern in Karlshorst geführt hatte, konkrete Verbesserungsvorschläge nur zu den familienrechtlichen Bestimmungen vorlegen. Für die SKK stand zumindest fest, daß das Gesetz überarbeitet und noch vor den anstehenden Wahlen im Oktober verabschiedet werden mußte ${ }^{159}$. Die Überarbeitung beschränkte sich offenbar im wesentlichen auf die Strukturierung des $\mathrm{Ge}$ setzentwurfs sowie auf einzelne Formulierungen. Inhaltliche Konkretisierungen waren nicht mehr möglich, da etwa die arbeitsmarktpolitischen Fördermaßnahmen Bestandteil des in Arbeit befindlichen Volkswirtschaftsplanes waren ${ }^{160}$.

150 Ebenda, Bl. 35.

151 SAPMO, NY 4090/563, B1. 65-67.

152 SAPMO, DY 30/IV 2/2/84, Bl. 14-16.

153 Ebenda, Bl. 17-24.

15+ SAPMO, NY 4090/563, Bl. 68, Notiz vom 9. 5. 1950 (o.Verf.).

155 Ebenda, Bl. 69f., hier Bl. 69, Aktenvermerk Heymanns vom 13. 5. 1950.

156 Ebenda, Bl. 70.

157 SAPMO, NY 4090/563, Bl. 80-89.

158 Die Position der SKK teilte Ministerpräsident Grotewohl bei einer Besprechung am 8. 6. 1950 mit. SAPMO, DY 30/IV 2/17/30, Bl. $92 \mathrm{f}$.

159 Ebenda.

160 SAPMO, NY 4090/563, Bl. 110 f., SED-Parteivorstand an das Sekretariat des Ministerpräsidenten (Tzschorn), eingegangen am 19.6. 1950. Dem Schreiben war cin von der SED-Führung nochmals überarbeiteter Gesetzentwurf beigefügt. Ebenda, Bl. 111-123. 
Die Zustimmung der SKK zum Gesetzesvorhaben lag Ende Juni 1950 vor $^{161}$. Ende August war schließlich der endgültige Entwurf fertig, der nunmehr die Bezeichnung „Gesetz über den Mutter- und Kinderschutz und die Rechte der deutschen Frau" trug ${ }^{162}$. Zu diesem Zeitpunkt stand bereits der zeitliche Ablauf bis zur Verabschiedung in der Volkskammer fest: Den handschriftlichen Notizen Grotewohls ist zu entnehmen, daß die Zustimmung des Politbüros am 5. September, des Ministerrates am 21. September und der Volkskammer am 4. Oktober geplant war ${ }^{163}$. Der erwähnte Entwurf wurde dem Politbüro zugeleitet und enthielt bereits die spätere Gliederung in vier größere Kapitel. Das erste Kapitel legte die staatlichen Hilfen für Mütter fest und enthielt einen konkreten Plan zum Ausbau von Kindergärten sowie von Kinderpolikliniken ${ }^{164}$. Erwähnenswert ist das darin enthaltene Verbot des Schwangerschaftsabbruchs, mit dem die bisherige weitgehend liberale Praxis, die vor allem dem Umstand der „Zusammenbruchsgesellschaft" der unmittelbaren Nachkriegszeit geschuldet war ${ }^{165}$, wieder rückgängig gemacht wurde und das bis ungefähr Anfang der siebziger Jahre Bestand haben sollte ${ }^{166}$. Das Gesetz über den Mutter- und Kinderschutz und die Rechte der Frau vom 27. September 1950 ließ nur noch die medizinische und eugenische Indikation als Begründung für eine legale Abtreibung $\mathrm{zu}^{167}$. Das zweite Kapitel faßte einige Bestimmungen zum Familienrecht zusammen und stand vor allem unter dem Motto der Gleichberechtigung von Mann und Frau. Dieses Ziel verfolgte auch das nachfolgende Kapitel, in dem die Teilnahme der Frauen in der betrieblichen Produktion proklamiert wurde ${ }^{168}$. Dabei sollte sich die berufliche Eingliederung nicht nur auf die Textil- und Konsumgüterindustrie beschränken, sondern auch Berufszweige erfassen, die bisher ausschließlich Männern vorbehalten waren. Der Entwurf vermied es jedoch, genaue Quoten sowie einen konkreten Zeitplan für die Durchführung dieser Forderung anzugeben. Des weiteren wurde das „Prinzip der gleichen Bezahlung für die gleiche Arbeit"169 erneut hervorgehoben und eine bevorzugte Ausbildung von Frauen in Facharbeiterberufen verlangt. Das letzte Kapitel regelte die Beteiligung von Frauen am staatlichen und gesellschaftlichen Leben und enthielt ebenfalls nur sehr vage gehaltene Bestimmungen ${ }^{170}$.

Das Politbüro des ZK der SED stimmte der Gesetzesvorlage am 5. September 1950 "grundsätzlich“ zu und beauftragte eine Kommission mit der Ausarbeitung der endgültigen Fassung des Gesetzestextes ${ }^{171}$. Der von Grotewohl skizzierte Zeitplan für die Verabschiedung des Gesetzes hatte im übrigen weiterhin Bestand.

161 Ebenda, Bl. 124, Notiz (o.Verf.) vom 23. 6. 1950.

162 Ebenda, Bl. 142-157.

163 Ebenda, Bl. 142.

164 SAPMO, NY 4090/563, Bl. 143-147.

165 Vgl. dazu Poutrus, Von den Massenvergewaltigungen zum Mutterschutzgesetz.

$166 \mathrm{Vgl}$. Harsch, Society, the State and Abortion in East Germany.

167 Poutrus, Von den Massenvergewaltigungen zum Mutterschutzgesetz, S. 190.

168 SAPMO, NY 4090/563, Bl. 150-153.

169 Ebenda, Bl. 150.

170 Ebenda, Bl. 153-156.

171 SAPMO, DY 30/IV 2/2/107, Bl. 21 f. Der Kommission gehörten an: Kulaczewski (ZK-Abt. Staatliche Verwaltung), Schaul (Regierungskanzlei), Käthe Selbmann (ZK-Abt. Frauen), Elli Schmidt (DFD), Käthe Kern (Ministerium für Arbeit und Gesundheitswesen), Friedel Malter (FDGB), Paul Peschke (Ministerium für Arbeit und Gesundheitswesen), Willi Georgino (Ministerium der Finanzen), Hans Nathan (Ministerium der Justiz) und Marta Chwalek (Magistrat Berlin). 
Die einberufene Kommission überarbeitete offenbar zügig den Entwurf, so daß dem SED-Führungsgremium bereits zur Sitzung am 12. September eine neue Fassung vorlag ${ }^{172}$, die mit zwei inhaltlichen Streichungen angenommen wurde ${ }^{173}$. Die Vorlage war mit den Ministerien der Finanzen und für Planung abgestimmt worden; letzteres hatte eine Reduzierung der Kinderkrippenplätze von 90000 auf 40000 sowie eine Erhöhung der Kindertagesplätze von 100000 auf 160000 erreichen können ${ }^{174}$. Auf Anraten des Ministeriums für Planung wurde außerdem aus Kostengründen zunächst auf die Gründung eines Forschungsinstitutes für Geburtshilfe verzichtet. $\mathrm{Da}$ das Politbüro am 26. September eine weitere Veränderung vornahm - dabei handelte es sich um eine Präzisierung der Arbeitszeitbestimmungen für Mütter von Kleinkindern -, verschob sich der Zeitplan etwas. Das Gesetz wurde schließlich von der Volkskammer am 27. September 1950 verabschiedet und trat am 1. Oktober in $\mathrm{Kraft}^{175}$.

Um die Auszahlung der einmaligen Beihilfen bzw. der staatlichen Unterstützungen, die erst mit der Geburt des dritten bzw. vierten Kindes einsetzen sollten, im einzelnen festzulegen, bedurfte es weiterer Ausführungsbestimmungen. Hierzu lag bereits Ende Oktober 1950 ein erster Entwurf von der Abteilung Mutter und Kind des Arbeitsministeriums vor ${ }^{176}$. In der Folge konnten sich jedoch die beiden beteiligten Ressorts, das Ministerium für Arbeit und Gesundheitswesen sowie das Ministerium der Finanzen, nicht über den Personenkreis einig werden, der in den Genuß der Leistungen kommen sollte. Während das Arbeitsministerium dafür plädierte, die Unterstützung für diejenigen Kinder auszuzahlen, die vor dem 1. Oktober 1950 geboren wurden und nach dem 30. September 1950 das 14. Lebensjahr vollendet hatten, erklärte das Finanzministerium, daß nur die nach dem 30. September geborenen Kinder Berücksichtigung finden sollten. Dahinter standen vermutlich finanzpolitische Erwägungen: Nach den Planungen des Arbeitsministeriums mußten ca. 120 Millionen DM pro Jahr im Haushalt veranschlagt werden, während das Finanzministerium auf eine Summe von rund 8 Millionen DM kam ${ }^{177}$. Da sich bei den Sozialversicherungskassen in der Zwischenzeit Anträge auf Auszahlung dieser Leistungen angehäuft hatten, die nicht bearbeitet werden konnten, wandte sich der Minister für Arbeit und Gesundheitswesen Steidle hilfesuchend an den Ministerpräsidenten und bat darum, eine „grundsätzliche Klärung der Zweifelsfragen" mit dem Finanzministerium herbeizuführen, zu der er sich offensichtlich nicht mehr in der Lage sah. Bereits zuvor waren alle Versuche des Arbeitsministeriums gescheitert, eine Entscheidung der SED-Führung zu erwirken ${ }^{178}$. Da sich eine Annäherung beider Positionen nicht abzeichnete, schaltete sich Anfang Dezember auch der DFD in die Debatte ein und schlug

172 SAPMO, NY 4090/563, Bl. 164-177, Gesetzentwurf vom 8. 9. 1950.

173 SAPMO, DY 30/IV 2/2/108, Bl. 79 und 91-104. Bei den Streichungen handelte es sich zum einen um Transferzahlungen an alleinerziehende Mütter sowie zum anderen um die Einführung eines bezahlten freien Haushaltstages. Beides entfiel ersatzlos.

174 SAPMO, NY 4090/563, Bl. 162 f., Vorlage der ZK-Abteilung Staatliche Verwaltung (Plenikowski) für das Politbüro (o.D.).

175 Gesetzblatt der DDR 1950, S. 1037-1041.

176 SAPMO, NY 4090/563, Bl. 205-208.

177 Ebenda, Bl. 196, Minister Steidle am 14. 11. 1950 an Ministerpräsident Grotewohl.

178 Ebenda, Bl. 198-204, Käthe Kern am 4. 11. 1950 an den SED-Vorsitzenden Otto Grotewohl. 
sich auf die Seite des Arbeitsministeriums. Die Frauenorganisation erinnerte Grotewohl in einem Schreiben daran, daß er „den Frauen selbst bei Anfrage“ zugesagt habe, es würden letztlich alle Neugeborenen unabhängig vom Geburtstermin berücksichtigt werden ${ }^{179}$. Der DFD wies darauf hin, daß „eine große Unruhe unter den Frauen entstanden" sei180; diese würden sich in Zuschriften oftmals auf die Zusage des Ministerpräsidenten berufen. Dagegen sah die Regierungskanzlei allem Anschein nach nicht die Dringlichkeit, zu einer raschen Entscheidung zu kommen, und bat die beiden Ministerien um eine erneute Stellungnahme ${ }^{181}$, obwohl die unterschiedlichen Positionen bereits deutlich geworden waren. Das Sekretariat des ZK befaßte sich kurz vor Jahresende mit der strittigen Frage, ohne aber eine Entscheidung zu treffen. Vielmehr wurde die Vorlage an das Politbüro weitergereicht und die Sozialversicherung angewiesen, mit der Leistungsauszahlung zu warten, bis die Durchführungsbestimmungen erlassen sind ${ }^{182}$. Der Konflikt wurde schließlich durch ein Machtwort der SKK entschieden, welche die Position des Finanzministeriums unterstützte und damit für einen eng begrenzten Personenkreis eintrat ${ }^{183}$, der von den im Gesetz vorgesehenen Beihilfen und Unterstützungen profitieren sollte. Somit konnte die Durchführungsbestimmung am 24. Januar 1951 veröffentlicht werden und erhielt rückwirkend zum 1. Oktober 1950 Gesetzeskraft ${ }^{184}$.

\section{Verordnung über die Neubildung und die Aufgaben der Arbeitsgerichte}

In der SBZ waren auf der Grundlage des SMAD-Befehls Nr. 23 vom 25. Januar 1946 sowie des Kontrollratsgesetzes Nr. 21 vom 30. März 1946 Arbeitsgerichte und Landesarbeitsgerichte errichtet worden. Dagegen war die Errichtung eines zonalen Arbeitsgerichtes als dritte Instanz, so wie es der Kontrollratsbefehl vorgesehen hatte, vorerst verschoben worden. Aus Sicht der Arbeitsverwaltung stellte die Arbeitsgerichtsbarkeit jedoch nur einen Bremsfaktor für die angestrebte Steuerung des Arbeitskräftepotentials dar. Schon frühzeitig wurde daher Kritik an den Urteilen einzelner Arbeitsrichter geübt. Nach Gründung der DDR griff das Ministerium für Arbeit und Gesundheitswesen den Gedanken wieder auf, eine weitere Gerichtsinstanz zu schaffen. Auf diese Weise erhoffte man sich im Ministerium, die unterschiedlich verlaufenden Eintwicklungen der Rechtsprechung

179 Ebenda, Bl. 213, DFD am 4. 12. 1950 an Ministerpräsident Grotewohl. Elli Schmidt wiederholte ihre Kritik gegenüber Grotewohl am 8.12.1950. Ebenda, Bl. $216 \mathrm{f}$.

180 Diesen Eindruck bestätigte auch das Arbeitsministerium. Vgl. SAPMO, NY 4090/563, Bl. 220f., Minister für Arbeit und Gesundheitswesen Steidle am 28.12. 1950 an den Chef der Regierungskanzlei. Nach Mitteilung des Zentralvorstandes der Sozialversicherung vom 6. 1. 1951 hatten nach einer Versammlung des DFD in einem Stahlwerk bei Merseburg 33 Frauen aus Protest gegen die vorgesehene Nichtauszahlung der Leistungen ihre DFD-Mitgliedsbücher zurückgegeben. Der Hauptgeschäftsführer des Zentralvorstandes Peschke erinnerte die Regierungskanzlei daran, daß die Durchführungsbestimmung „so schnell wie möglich erlassen werden“ müsse. Ebenda, Bl. 253.

181 SAPMO, NY 4090/563, Bl. 215, Staatssekretär Geyer am 7. 12. 1950 an die Ministerien der Finanzen und für Gesundheitswesen.

182 SAPMO, DY 30/J IV 2/3/162, Bl. 1, Protokoll der Sitzung des Sekretariats des ZK vom 22. 12. 1950.

183 SAPMO, NY 4090/563, Bl. 255, Ministerium der Finanzen (Staatssekretär Rumpf) am 29. 1.1951 an Ministerpräsident Grotewohl.

184 Gesetzblatt der DDR 1951, S. $37 \mathrm{f}$. 
auf diesem Gebiet in den einzelnen Ländern wieder stärker zusammenfassen zu können und eine Vereinheitlichung zu erzielen ${ }^{185}$. Dazu legte die Abteilung Arbeitsrecht Anfang April 1951 einen ersten Entwurf vor ${ }^{186}$. Demzufolge sollten beim Obersten Arbeitsgericht Senate für Arbeitsrechts- und Sozialversicherungsrechtsstreitfälle gebildet werden, die jeweils mit einem Vorsitzenden, zwei hauptamtlichen und zwei ehrenamtlichen Arbeitsrichtern zu besetzen waren. Die Mitglieder des Obersten Arbeitsgerichts wurden laut Gesetzentwurf von der Volkskammer auf Vorschlag der DDR-Regierung und des FDGB-Bundesvorstandes gewählt und abberufen. Qualifikationsvoraussetzung sollte der erfolgreiche Abschluß der sogenannten großen juristischen Staatsprüfung oder die Absolvierung einer Volksrichterschule sein. Darüber hinaus konnten auch „hervorragende Praktiker oder Wissenschaftler" aus dem Arbeits- bzw. Sozialversicherungsrecht berufen werden. Der Entwurf sah vor, daß das Oberste Arbeitsgericht dem Arbeitsministerium unterstellt wurde und „für die Verhandlung und Entscheidung über den Antrag auf Kassation rechtskräftiger arbeits- und sozialversicherungsrechtlicher Entscheidungen zuständig" war ${ }^{187}$. Antragsberechtigt waren allerdings nur einzelne Minister und Staatssekretäre, die Ministerpräsidenten der Länder sowie der FDGB-Bundesvorstand ${ }^{188}$. Ausgeschlossen blieben Anträge auf Einleitung eines Revisionsverfahrens durch Einzelpersonen, die somit nur die Arbeitsund Landesarbeitsgerichte anrufen konnten. Dadurch war die Möglichkeit für staatliche Funktionsträger eröffnet worden, Entscheidungen der ersten und zweiten Instanz wieder rückgängig machen zu können. In der Folgezeit griffen weder der DDR-Ministerrat noch der FDGB-Bundesvorstand diesen Vorschlag auf, so daß der Entwurf rasch wieder in der Versenkung verschwand.

Strukturelle Veränderungen erfolgten erst 1953, nachdem ein Jahr zuvor die Länder aufgelöst worden waren und die Bildung der Bezirke auch langfristige Folgen für die Arbeitsgerichtsbarkeit in der DDR hatte. Die Ausarbeitung einer Verordnung "über die Neugliederung und die Aufgaben der Arbeitsgerichte" verzögerte sich etwas ${ }^{189}$, so daß die DDR-Regierung erst am 30. April 1953 einen entsprechenden Beschluß fassen konnte ${ }^{190}$, nachdem das Sekretariat des ZK dem Entwurf zehn Tage vorher seine Zustimmung gegeben hatte ${ }^{191}$. Die verabschiedete Verordnung legte fest, daß sich die Arbeitsgerichte in Bezirks- und Kreisarbeitsgerichte gliedern ${ }^{192}$. Das Oberste Gericht sollte dagegen als Kassationsgericht auch für die Arbeitsrechtsfälle zuständig sein. Somit hatte sich das Vorhaben des Arbeitsministeriums von 1951, ein Oberstes Arbeitsgericht einzurichten, endgültig erübrigt. Eine erhebliche qualitative Veränderung brachte die zeitgleich beschlossene Verordnung über die Bildung von Kommissionen zur Beseitigung von Arbeitsstreitfällen in den volkseigenen und ihnen gleichgestellten Betrieben und

\footnotetext{
$185 \mathrm{BAB}, \mathrm{DQ} 2 / 1798$, Begründung der Abt. Arbeitsrecht zum Gesetzentwurf vom 7. 4. 1951, S. 1.

186 Ebenda, I. Entwurf der Abt. Arbeitsrecht vom 6. 4. 1951.

187 Ebenda, S. $2(\$ 6)$.

188 Ebenda, S. $3(\$ 8)$.

189 Vgl. BAB, DC 20 I/3-185, Bl. 162, Vizepräsident des Obersten Gerichts am 10. 3. 1953 an Staatssekretärin Malter; cbenda, Bl. 196-198, Generalstaatsanwalt am 13.3.1953 an Minister Chwalek. 190 BAB, DC $20 \mathrm{I} / 3-185, \mathrm{Bl} .7$.

191 SAPMO, DY 30/J IV 2/3/377, Bl. 3, Protokoll der Sitzung des Sekretariats des ZK am 20. 4. 1953.

$192 \mathrm{BAB}, \mathrm{DC} 20 \mathrm{I} / 3-185, \mathrm{Bl} .21-27$.
} 
in den Verwaltungen, den sogenannten Konfliktkommissionen ${ }^{193}$. Obwohl die Arbeitsgerichte nicht vollständig beseitigt wurden, bedeutete der Auf- und Ausbau der Konfliktkommissionen eine allmähliche Aushöhlung der bisherigen Arbeitsgerichtsbarkeit ${ }^{194}$. Beide Verordnungen erschienen am 13. Mai 1953 im Gesetzblatt der DDR ${ }^{195}$ und hoben die beiden Befehle der sowjetischen Besatzungsmacht sowie des Alliierten Kontrollrates auf. 